\title{
Chiral N-Heterocyclic Carbene Catalyzed, Enantioselective Oxodiene Diels-Alder Reactions with Low Catalyst Loadings
}

\author{
Ming He, Gerson J. Uc, and Jeffrey W. Bode* \\ Department of Chemistry and Biochemistry, University of California, \\ Santa Barbara, CA 93106-9510
}

\section{Supporting Information}

General Methods. All reactions utilizing air- or moisture-sensitive reagents were performed in dried glassware under an atmosphere of dry argon. $\mathrm{CH}_{2} \mathrm{Cl}_{2}$ was distilled over $\mathrm{CaH}_{2}$. EtOAc, toluene and THF were dried by passage over activated alumina under Ar atmosphere. All aldehydes were purified by distillation prior to use. Triethylamine $\left(\mathrm{NEt}_{3}\right)$ was distilled from $\mathrm{CaH}_{2}$. Other reagents were used without further purification. Thin layer chromatography (TLC) was performed on Merck precoated plates (silica gel $60 \mathrm{~F}_{254}$, Art 5715, $0.25 \mathrm{~mm}$ ) and were visualized by fluorescence quenching under UV light or by staining with phosphomolybdic acid. Silica-gel preparative thin-layer chromatography (PTLC) was performed using plates prepared from Merck Kieselgel $60 \mathrm{PF}_{254}$ (Art 7747). Column chromatography was performed on E. Merck Silica Gel 60 (230-400 Mesh) using a forced flow of 0.5-1.0 bar. ${ }^{1} \mathrm{H}$ NMR (400 MHz) and ${ }^{13} \mathrm{C}$ NMR (100 MHz) were measured on a Varian Unity 400 spectrometer. The peaks labeled with * indicate the minor diastereomers (trans). Chemical shifts are expressed in parts per million (PPM) downfield from residual solvent peaks and coupling constants are reported as Hertz (Hz). Splitting patterns are indicated as follows: br, broad; s, singlet; d, doublet; t, triplet; q, quartet; m, multiplet. Infrared (IR) spectra were recorded on a JASCO FT/IR-430 spectrophotometer and are reported as wavenumber $\left(\mathrm{cm}^{-1}\right)$. Optical rotations were measured on a Jasco DIP-1000 polarimeter operating at the sodium $\mathrm{D}$ line with a $100 \mathrm{~mm}$ path length cell, and are reported as follows: $[\alpha]^{\mathrm{T}}$ (concentration $(\mathrm{g} / 100 \mathrm{ml})$, solvent).

HPLC Conditions. Column, Daicel Chiralpak AS-H, (4.6 x 250mm) Eluent: hexanes/i-PrOH. Flow Rate $1.0 \mathrm{~mL} / \mathrm{min}$. Detection: $254 \mathrm{~nm}$. Column, Daicel Chiralpak AD-H, (4.6 x250mm) Eluent: hexanes/iPrOH. Flow Rate $1.0 \mathrm{~mL} / \mathrm{min}$. Detection: $254 \mathrm{~nm}$. Column, Daicel Chiralpak OD-H, (4.6 x250mm) Eluent: hexanes/iPrOH. Flow Rate $1.0 \mathrm{~mL} / \mathrm{min}$. Detection: $254 \mathrm{~nm}$. 
Preparative scale enantioselective oxodiene Diels-Alder reactions.

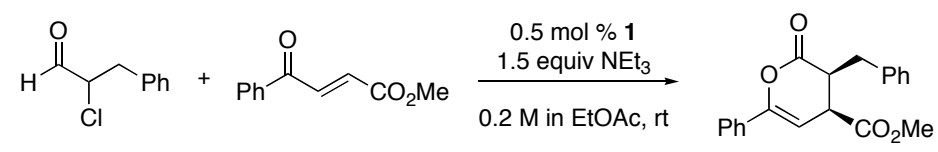

Into an oven dried $200 \mathrm{ml}$ round-bottom flask was added 2-chloro-hydrocinnamaldehyde $(6.40 \mathrm{~g}$, $38.0 \mathrm{mmol}, 1.6$ equiv) and (E)-methyl 4-oxo-4-phenylbut-2-enoate (4.51 g, $23.7 \mathrm{mmol}, 1.0$ equiv). To this mixture was added $120 \mathrm{ml}$ EtOAc $(0.2 \mathrm{M})$, followed by addition of triazolium salt $^{1} 1$ (44 mg, $\left.0.12 \mathrm{mmol}, 0.5 \mathrm{~mol} \%\right)$ and $\mathrm{NEt}_{3}(5.0 \mathrm{ml}, 36 \mathrm{mmol}, 1.5$ equiv). The white $\mathrm{NEt}_{3} \bullet \mathrm{HCl}$ salt precipitated in 2 5 minutes. The reaction was stirred under Ar for 2 hours. Water was added to the vial and the mixture was extracted with EtOAc $(3 \times 100 \mathrm{ml})$. The combined EtOAc extract was washed with brine and dried over anhydrous $\mathrm{NaSO}_{4}$. The solution was concentrated under reduced pressure, and the resulting pale yellow solid was recrystallized from 10:1 hexane/EtOAc to afford $5.29 \mathrm{~g}$ white solid as a single diasteromer. The residue was purified by silica gel chromatography (10:1 hexane/EtOAc) to afford another $2.19 \mathrm{~g}$ as a 4:1 mixture of diastereomers. The combined yield was $98 \%$ with 15:1 dr (7.48 g, $23.2 \mathrm{mmol})$.

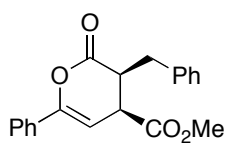

(3S,4S)-Methyl 3-benzyl-6-phenyl-2-oxo-3,4-dihydro-2H-pyran-4-carboxylate. (Table 1 , entry 3) $[\alpha]_{\mathrm{D}}{ }^{20}\left(\mathrm{c} 1.20, \mathrm{CHCl}_{3}\right)=+151.2 ; \mathrm{mp}=105-106{ }^{\circ} \mathrm{C} ;{ }^{1} \mathrm{H} \mathrm{NMR}\left(400 \mathrm{MHz}, \mathrm{CDCl}_{3}\right) \delta 7.63-$ $7.61(\mathrm{~m}, 2 \mathrm{H}), 7.39-7.27(\mathrm{~m}, 6 \mathrm{H}), 7.27-7.17(\mathrm{~m}, 2 \mathrm{H}), 5.81(\mathrm{~d}, 1 \mathrm{H}, J=7.0 \mathrm{~Hz}), 3.76(\mathrm{~s}, 3 \mathrm{H}), 3.55$ $(\mathrm{dd}, 1 \mathrm{H}, J=14.3,4.6 \mathrm{~Hz}), 3.31-3.28(\mathrm{~m}, 1 \mathrm{H}), 3.04-2.99(\mathrm{~m}, 1 \mathrm{H}), 2.76(\mathrm{dd}, 1 \mathrm{H}, J=14.3,10.1$ $\mathrm{Hz}) ;{ }^{13} \mathrm{C} \mathrm{NMR}\left(100 \mathrm{MHz}, \mathrm{CDCl}_{3}\right) \delta 170.9,168.9,152.6,138.2,131.8,129.8,129.2,129.1$, 128.8, 127.1, 125.0, 98.1, 52.8, 42.8, 40.6, 33.4; IR (thin film) v 3061, 3028, 2952, 1777, 1733, 1496, 1448, 1173, $1069 \mathrm{~cm}^{-1}$; HRMS (ESI) calcd for $\mathrm{C}_{20} \mathrm{H}_{18} \mathrm{O}_{4}[\mathrm{M}+\mathrm{Na}]^{+}$345.1097, found 345.1094; >99\% ee $(3 S, 4 S)$-isomer as determined by HPLC (AD-H, 9:1 hexanes/i-PrOH), $t_{\mathrm{r}}(3 S$, $4 S)=32.4 \min , t_{\mathrm{r}}(3 R, 4 R)=20.3 \min$.

(1) He, M.; Struble, J. R.; Bode, J. W. J. Am. Chem. Soc. 2006, 128, 8418-8420 
From $(\mathbf{R}, \mathbf{S})$ Catalyst:
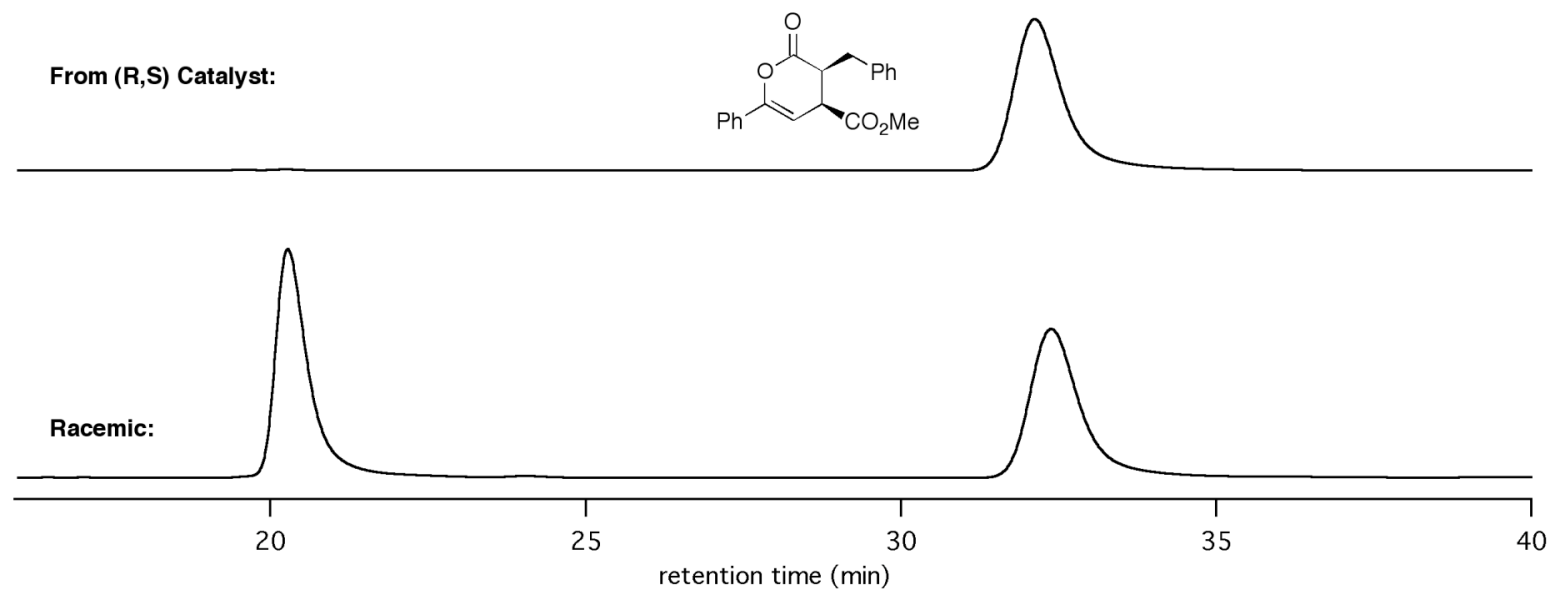

General procedure for NHC-catalyzed, enantioselective oxodiene Diels-Alder reactions.

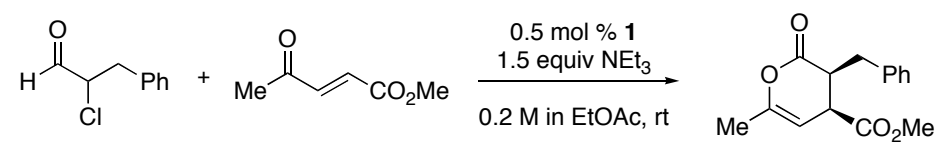

The reaction of 2-chloro-hydrocinnamaldehyde and (E)-methyl 4-oxopent-2-enoate is representative: Into an oven dried $4.0 \mathrm{~mL}$ vial was added 2-chloro-hydrocinnamaldehyde (146.0 mg, $0.87 \mathrm{mmol}, 1.6$ equiv), (E)-methyl 4-oxopent-2-enoate (77.8 mg, $0.54 \mathrm{mmol}, 1.0$ equiv) and triazolium salt $1(1.0 \mathrm{mg}, 0.5 \mathrm{~mol} \%)$. To this mixture was added $2.7 \mathrm{~mL}$ EtOAc $(0.2 \mathrm{M})$, followed by $\mathrm{NEt}_{3}\left(112 \mu \mathrm{L}, 0.82 \mathrm{mmol}, 1.5\right.$ equiv). The vial was capped and the white $\mathrm{NEt}_{3} \bullet \mathrm{HCl}$ salt precipitated in 2 5 minutes. The mixture was stirred at $\mathrm{rt}$ for $6 \mathrm{~h}$. Water was added to the vial and the mixture was extracted with EtOAc $(2 \times 5 \mathrm{ml})$. The combined EtOAc extract was washed with brine and dried over anhydrous $\mathrm{NaSO}_{4}$. The solution was concentrated under reduced pressure, and the residue was purified by silica gel chromatography (10:1 hexane/EtOAc) to afford the product as a single diastereomer (123.2 mg, 88\% yield). 


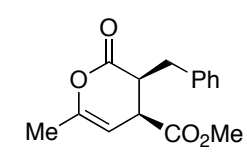

(3S,4S)-Methyl 3-benzyl-6-methyl-2-oxo-3,4-dihydro-2H-pyran-4-carboxylate (Table 1, entry 1). Prepared according to the general procedure from 2-chloro-hydrocinnamaldehyde and (E)-methyl 4-oxopent-2-enoate using $0.5 \mathrm{~mol} \mathrm{\%} 1$ as the catalyst in $88 \%$ yield as a single diasteromer (colorless oil). $[\alpha]_{\mathrm{D}}{ }^{20}\left(\mathrm{c} 1.10, \mathrm{CHCl}_{3}\right)=+218.7 ;{ }^{1} \mathrm{H} \mathrm{NMR}\left(400 \mathrm{MHz}, \mathrm{CDCl}_{3}\right) \delta 7.32-$ $7.23(\mathrm{~m}, 3 \mathrm{H}), 7.12(\mathrm{~d}, 2 \mathrm{H}, J=7.2 \mathrm{~Hz}), 5.02(\mathrm{~d}, 1 \mathrm{H}, J=7.2 \mathrm{~Hz}), 3.73(\mathrm{~s}, 3 \mathrm{H}), 3.48(\mathrm{dd}, 1 \mathrm{H}, J=$ $14.3,4.6 \mathrm{~Hz}), 3.02(\mathrm{t}, 1 \mathrm{H}, J=6.0 \mathrm{~Hz}), 2.89-2.84(\mathrm{~m}, 1 \mathrm{H}), 2.66(\mathrm{dd}, 1 \mathrm{H}, J=14.3,10.1 \mathrm{~Hz}), 1.89$ $(\mathrm{s}, 3 \mathrm{H}) ;{ }^{13} \mathrm{C} \mathrm{NMR}\left(100 \mathrm{MHz}, \mathrm{CDCl}_{3}\right) \delta 171.3,169.3,152.6,138.3,129.2,129.0,127.0,98.7$, 52.6, 42.8, 40.3, 33.4, 19.0; IR (thin film) v 3062, 2954, 1780, 1732, 1496, 1435, 1362, $1164 \mathrm{~cm}^{-}$ ${ }^{1}$; HRMS (ESI) calcd for $\mathrm{C}_{15} \mathrm{H}_{16} \mathrm{O}_{4}[\mathrm{M}+\mathrm{Na}]^{+}$283.0940, found 283.0944; $99 \%$ ee $(3 S, 4 S)$-isomer as determined by HPLC (AD-H, 9:1 hexanes/i-PrOH), $t_{\mathrm{r}}(3 S, 4 S)=12.0 \mathrm{~min}, t_{\mathrm{r}}(3 R, 4 R)=9.40$ $\min$.

From $(\mathbf{R}, \mathrm{S})$ Catalyst:
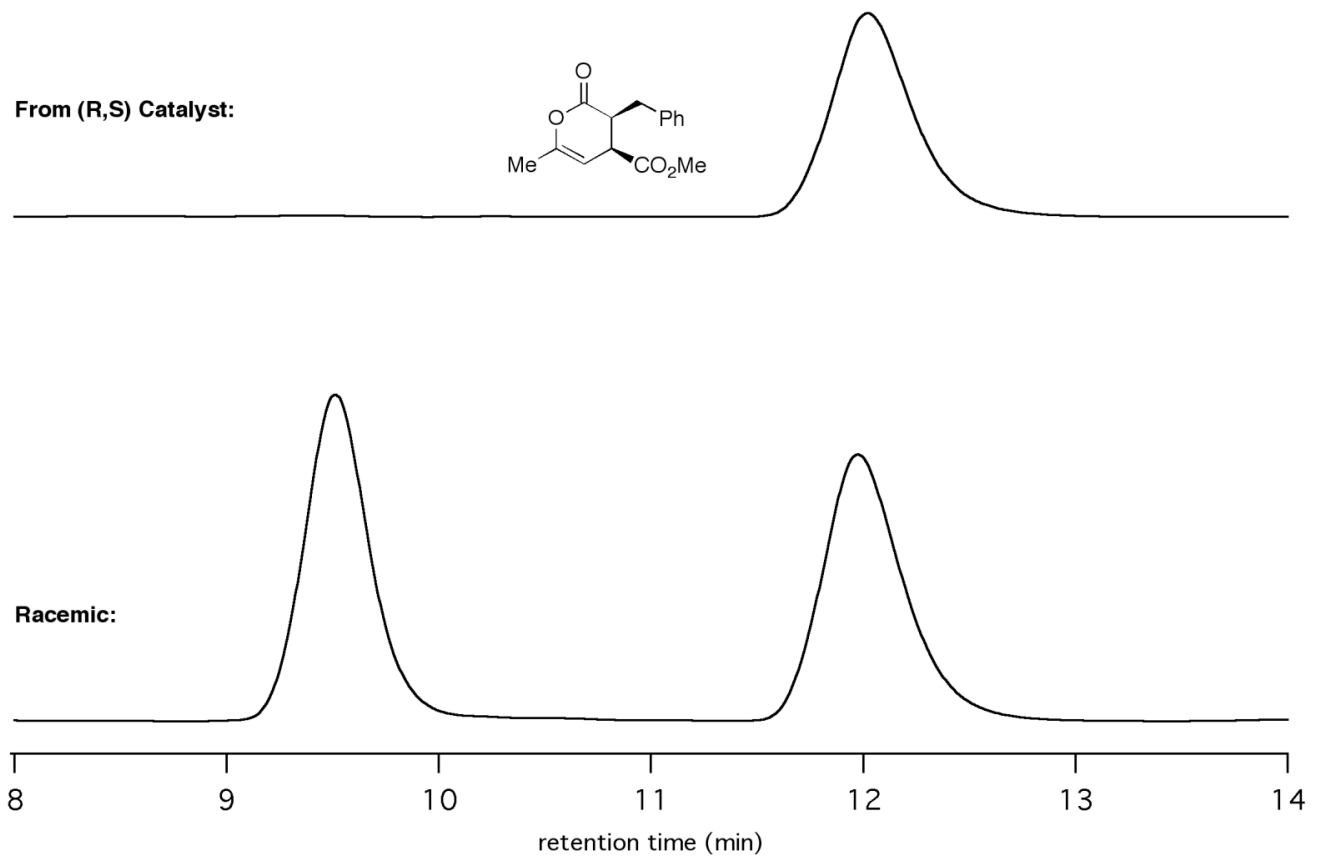


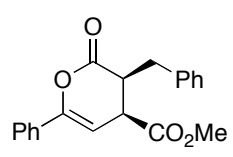

(3S,4S)-Methyl 3-benzyl-6-phenyl-2-oxo-3,4-dihydro-2H-pyran-4-carboxylate (Table 1, entry

2). Prepared according to the general procedure from 2-chloro-hydrocinnamaldehyde and $(E)$ methyl 4-oxo-4-phenylbut-2-enoate using $0.5 \mathrm{~mol} \mathrm{\%} \mathbf{1}$ as the catalyst in $91 \%$ yield as a 8:1 mixture of diastereomers (white solid). $[\alpha]_{\mathrm{D}}{ }^{20}\left(\mathrm{c} 1.16, \mathrm{CHCl}_{3}\right)=+130.4 ; \mathrm{mp}=103-106{ }^{\circ} \mathrm{C} ;{ }^{1} \mathrm{H}$ NMR (400 MHz, $\left.\mathrm{CDCl}_{3}\right) \delta$ 7.67-7.61 (m, 2H), 7.41-7.27 (m, 6H), 7.27-7.17 (m, 2H), $5.82(\mathrm{~d}$, $1 \mathrm{H}, J=7.0 \mathrm{~Hz}), 5.75^{*}(\mathrm{~d}, 1 \mathrm{H}, J=5.7 \mathrm{~Hz}), 3.77(\mathrm{~s}, 3 \mathrm{H}), 3.69 *(\mathrm{~s}, 3 \mathrm{H}), 3.61-3.58 *(\mathrm{~m}, 1 \mathrm{H}), 3.56$ $(\mathrm{dd}, 1 \mathrm{H}, J=14.3,4.6 \mathrm{~Hz}), 3.42-3.37 *(\mathrm{~m}, 1 \mathrm{H}), 3.32-3.28(\mathrm{~m}, 1 \mathrm{H}), 3.17 *(\mathrm{dd}, 1 \mathrm{H}, J=13.8,5.7$ $\mathrm{Hz}), 3.04-2.99(\mathrm{~m}, 1 \mathrm{H}), 2.92^{*}(\mathrm{dd}, 1 \mathrm{H}, J=13.8,9.2 \mathrm{~Hz}), 2.77(\mathrm{dd}, 1 \mathrm{H}, J=14.3,10.1 \mathrm{~Hz}) ;{ }^{13} \mathrm{C}$ NMR (100 MHz, $\left.\mathrm{CDCl}_{3}\right) \delta 170.8,168.9,152.6,138.2,131.8,129.8,129.4,129.2,129.0,128.8$, 127.4, 127.1, 125.0, 98.1, 95.9, 52.9, 52.8, 42.8, 42.3, 41.2, 40.6, 35.5, 33.4; IR (thin film) $v$ 3061, 3028, 2952, 1777, 1733, 1496, 1448, 1173, $1069 \mathrm{~cm}^{-1}$; HRMS (ESI) calcd for $\mathrm{C}_{20} \mathrm{H}_{18} \mathrm{O}_{4}$ $[\mathrm{M}+\mathrm{Na}]^{+} 345.1097$, found $345.1094 ;>99 \%$ ee $(3 S, 4 S)$-isomer as determined by HPLC (AD-H, 9:1 hexanes/i-PrOH $), t_{\mathrm{r}}(3 S, 4 S)=32.4 \mathrm{~min}, t_{\mathrm{r}}(3 R, 4 R)=20.3 \mathrm{~min}$.

From (R,S) Catalyst:
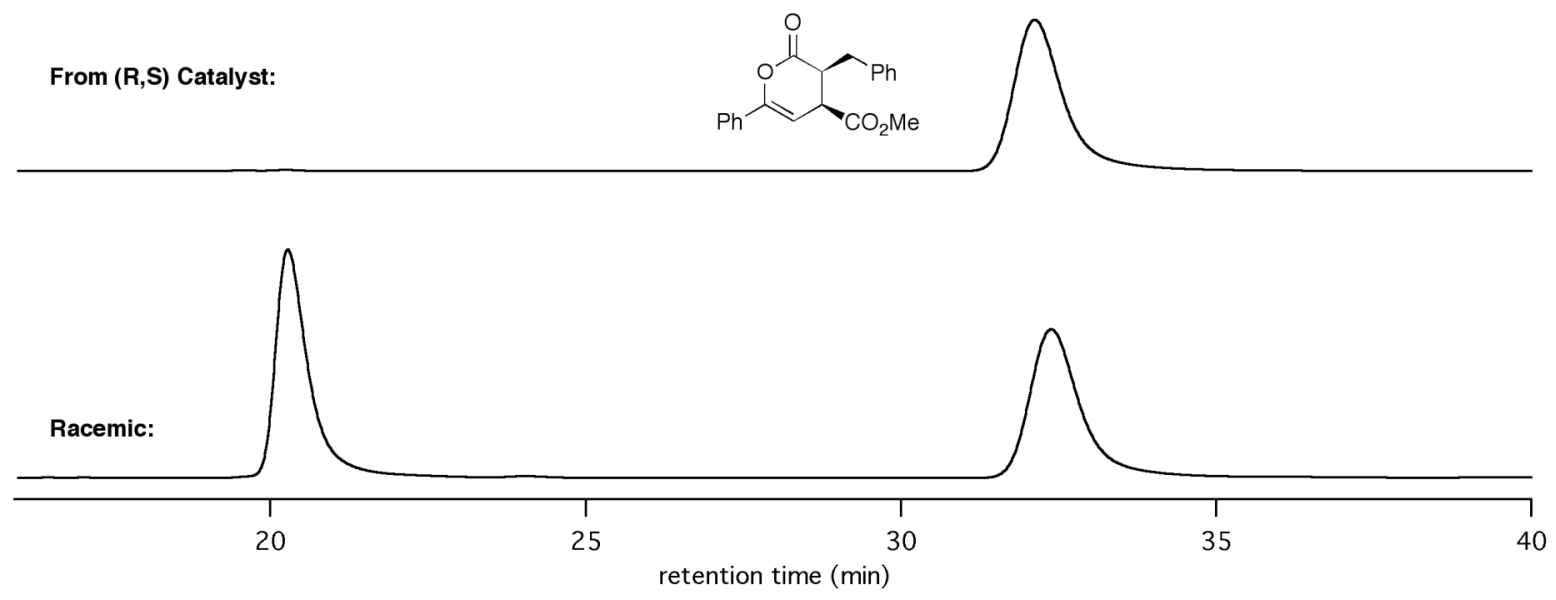


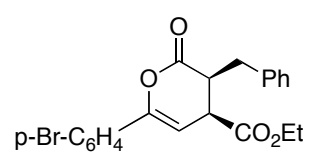

(3S,4S)-Methyl 3-benzyl-6-(4-bromophenyl)-2-oxo-3,4-dihydro-2H-pyran-4-carboxylate (Table 1, entry 4). Prepared according to the general procedure from 2-chlorohydrocinnamaldehyde and (E)-methyl 4-oxo-4-(4-bromophenyl)but-2-enoate using 0.5 mol \% 1 as the catalyst in $80 \%$ yield as a $6: 1$ mixture of diastereomers (white solid). $[\alpha]_{\mathrm{D}}{ }^{20}$ (c 1.23 , $\left.\mathrm{CHCl}_{3}\right)=+112.1 ; \mathrm{mp}=139-141{ }^{\circ} \mathrm{C} ;{ }^{1} \mathrm{H}$ NMR $\left(400 \mathrm{MHz}, \mathrm{CDCl}_{3}\right) \delta$ 7.53-7.47 (m, 4H), 7.35$7.25(\mathrm{~m}, 5 \mathrm{H}), 7.19-7.17(\mathrm{~m}, 2 \mathrm{H}), 5.81(\mathrm{~d}, 1 \mathrm{H}, J=7.1 \mathrm{~Hz}), 5.74^{*}(\mathrm{~d}, 1 \mathrm{H}, J=5.6 \mathrm{~Hz}), 4.23(\mathrm{q}$, $2 \mathrm{H}, J=7.0 \mathrm{~Hz}), 4.13^{*}(\mathrm{q}, 2 \mathrm{H}, J=7.2 \mathrm{~Hz}), 3.55(\mathrm{dd}, 1 \mathrm{H}, J=14.4,4.7 \mathrm{~Hz}), 3.40-3.35^{*}(\mathrm{~m}, 1 \mathrm{H})$, 3.27-3.24* (m. 1H), 3.25 (dd, 1H, $J=7.1,5.8 \mathrm{~Hz}), 3.14 *(\mathrm{dd}, 1 \mathrm{H}, J=13.8,5.7 \mathrm{~Hz}), 3.03-2.97$ $(\mathrm{m}, 1 \mathrm{H}), 2.91 *(\mathrm{dd}, 1 \mathrm{H}, J=13.8,8.8 \mathrm{~Hz}), 2.77(\mathrm{dd}, 1 \mathrm{H}, J=14.3,10.0 \mathrm{~Hz}), 1.30(\mathrm{t}, 3 \mathrm{H}, J=7.0$ $\mathrm{Hz}), 1.24^{*}(\mathrm{t}, 3 \mathrm{H}, J=7.2 \mathrm{~Hz}) ;{ }^{13} \mathrm{C} \mathrm{NMR}\left(100 \mathrm{MHz}, \mathrm{CDCl}_{3}\right) \delta 170.2,168.7,151.7,138.1,131.9$, $130.9,1294,129.2,129.0,127.4,127.1,126.5,124.0,98.7,96.7,66.1,62.1,61.9,42.8,42.2$, 41.5, 40.7, 35.5, 33.3, 15.5, 14.3, 14.2; IR (thin film) v 3087, 3027, 2980, 1778, 1731, 1489, 1455, 1176, 1069, $1009 \mathrm{~cm}^{-1}$; HRMS (ESI) calcd for $\mathrm{C}_{21} \mathrm{H}_{19} \mathrm{BrO}_{4}[\mathrm{M}+\mathrm{Na}]^{+} 437.0358$, found 437.0359; $99 \%$ ee $(3 S, 4 S)$-isomer as determined by HPLC (AD-H, 9:1 hexanes/i-PrOH), $t_{\mathrm{r}}(3 S$, $4 S)=57.6 \min , t_{\mathrm{r}}(3 R, 4 R)=25.4 \mathrm{~min}$.

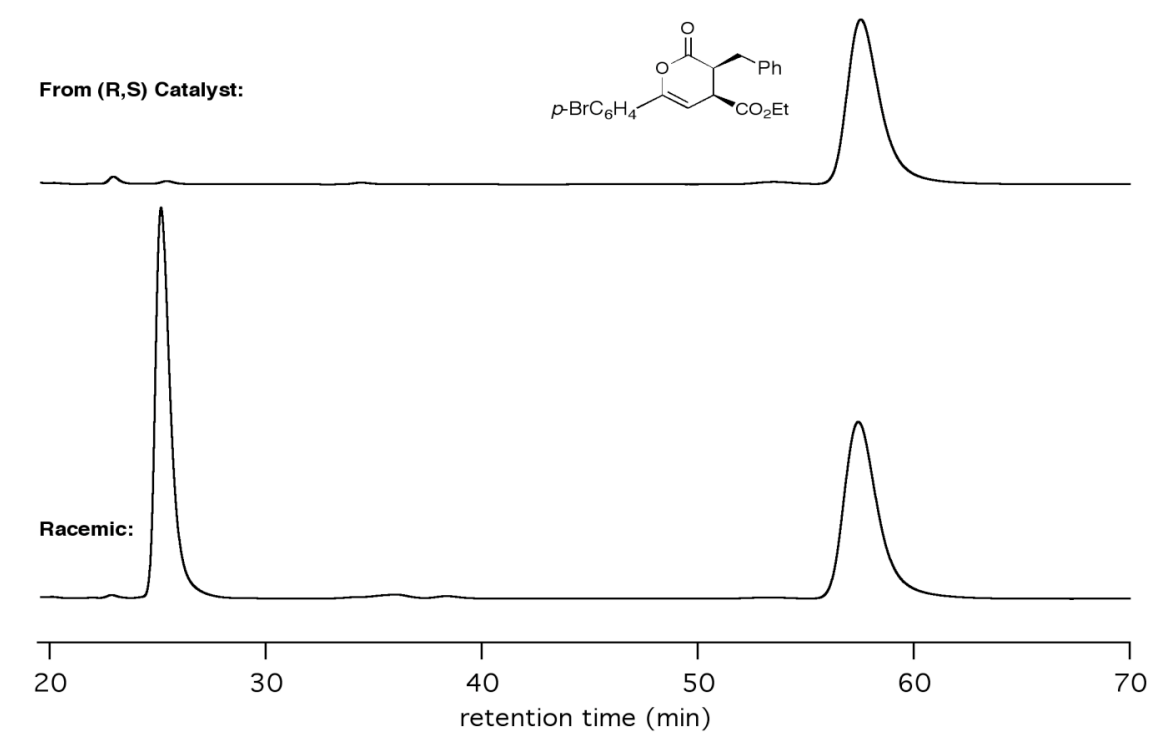

Page S6 


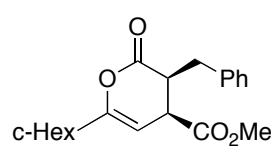

(3S,4S)-Methyl 3-benzyl-6-cyclohexyl-2-oxo-3,4-dihydro-2H-pyran-4-carboxylate (Table 1 , entry 5). Prepared according to the general procedure from 2-chloro-hydrocinnamaldehyde and (E)-methyl 4-oxo-4-cyclohexylbut-2-enoate using $0.5 \mathrm{~mol} \% \mathbf{1}$ as the catalyst in $76 \%$ yield as a single diasteromer (white solid). $[\alpha]_{\mathrm{D}}{ }^{20}\left(\mathrm{c} 1.06, \mathrm{CHCl}_{3}\right)=+178.6 ; \mathrm{mp}=87-89{ }^{\circ} \mathrm{C} ;{ }^{1} \mathrm{H} \mathrm{NMR}(400$ $\left.\mathrm{MHz}, \mathrm{CDCl}_{3}\right) \delta$ 7.32-7.25 (m, 3H), 7.15-7.13 (m, 2H), $4.98(\mathrm{~d}, 1 \mathrm{H}, J=6.9 \mathrm{~Hz}), 3.73(\mathrm{~s}, 3 \mathrm{H})$, $3.47(\mathrm{dd}, 1 \mathrm{H}, J=14.3,4.8 \mathrm{~Hz}), 3.06$ (t. $1 \mathrm{H}, J=6.3 \mathrm{hz}), 2.86-2.81(\mathrm{~m}, 1 \mathrm{H}), 2.67(\mathrm{dd}, 1 \mathrm{H}, J=$ 14.3, 10.1 Hz), 2.06-2.03 (m, 1H), 1.88-1.83 (m, 5H), 1.28-1.13 (m, 5H); ${ }^{13} \mathrm{C} \mathrm{NMR} \mathrm{(100} \mathrm{MHz,}$ $\left.\mathrm{CDCl}_{3}\right) \delta 171.4,169.6,170.3,138.3,129.1,128.9,127.0,96.2,52.6,43.0,41.0,40.0,33.4,30.2$, 30.0, 26.1, 26.0, 25.9; IR (thin film) v 3086, 3027, 2929, 2854, 1773, 1737, 1452, 1436, 1155 , $1056 \mathrm{~cm}^{-1}$; HRMS (ESI) calcd for $\mathrm{C}_{20} \mathrm{H}_{24} \mathrm{O}_{4}[\mathrm{M}+\mathrm{Na}]^{+} 351.1566$, found 351.1574; 86\% ee (3S, $4 S)$-isomer as determined by HPLC (OD-H, 9:1 hexanes/i-PrOH), $t_{\mathrm{r}}(3 S, 4 S)=8.5 \mathrm{~min}, t_{\mathrm{r}}(3 R, 4 R)$ $=7.7 \mathrm{~min}$.
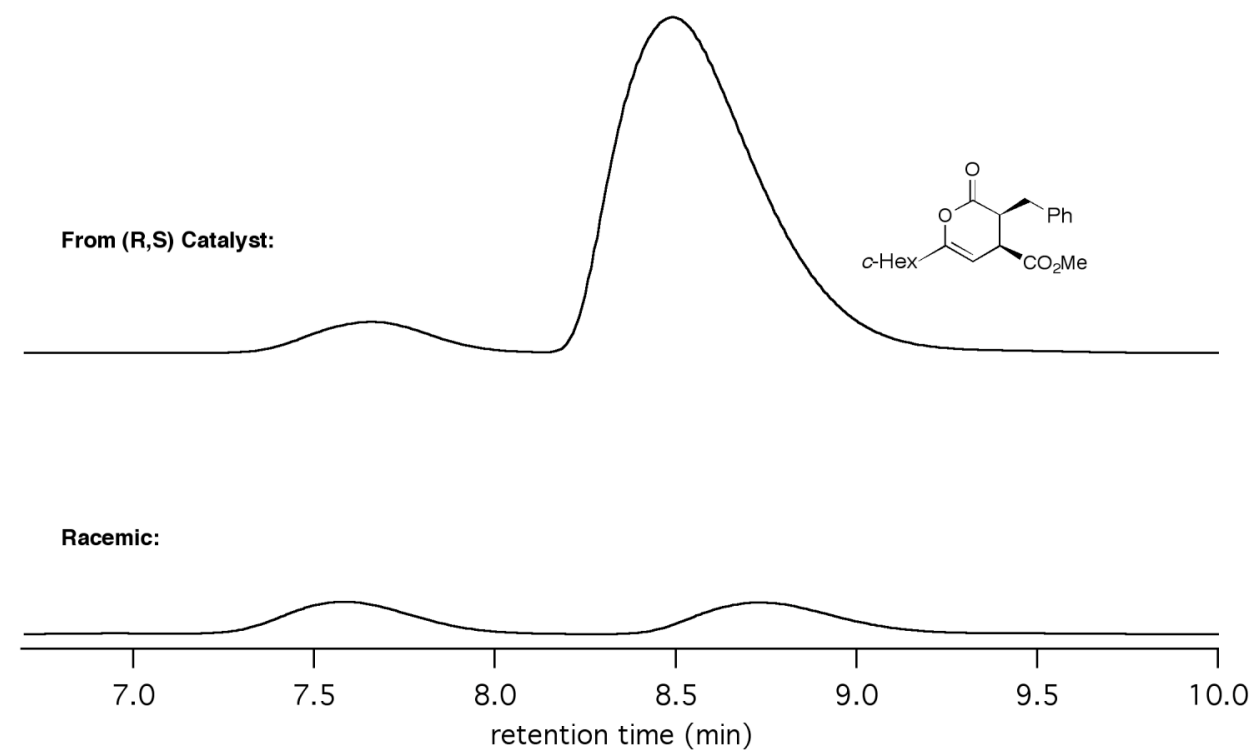


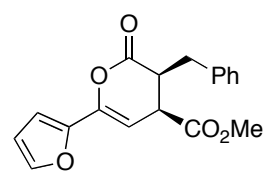

(3S,4S)-Methyl 3-benzyl-6-furanyl-2-oxo-3,4-dihydro-2H-pyran-4-carboxylate (Table 1, entry 6). Prepared according to the general procedure from 2-chloro-hydrocinnamaldehyde and (E)-methyl 4-oxo-4-furanylbut-2-enoate using $0.5 \mathrm{~mol} \% 1$ as the catalyst in $94 \%$ yield as an 8:1 mixture of diastereomers (brown oil). $[\alpha]_{\mathrm{D}}^{20}\left(\mathrm{c} 1.03, \mathrm{CHCl}_{3}\right)=+119.5 ;{ }^{1} \mathrm{H} \mathrm{NMR}(400 \mathrm{MHz}$, $\left.\mathrm{CDCl}_{3}\right) \delta 7.41-7.29(\mathrm{~m}, 4 \mathrm{H}), 7.18-7.16(\mathrm{~m}, 2 \mathrm{H}), 6.67(\mathrm{~d}, 1 \mathrm{H}, J=3.4 \mathrm{~Hz}), 6.47 *(\mathrm{dd}, 1 \mathrm{H}, J=3.2$, $2.0 \mathrm{~Hz}), 6.45(\mathrm{dd}, 1 \mathrm{H}, J=3.2,1.6 \mathrm{~Hz}), 5.74(\mathrm{~d}, 1 \mathrm{H}, J=7.0 \mathrm{~Hz}), 5.70 *(\mathrm{~d}, 1 \mathrm{H}, J=6.0 \mathrm{~Hz}), 3.77$ (s, 3H), 3.68* (s, 3H), $3.55(\mathrm{dd}, 1 \mathrm{H}, J=14.3,4.7 \mathrm{~Hz}), 3.52-3.46^{*}(\mathrm{~m}, 1 \mathrm{H}), 3.40-3.35^{*}(\mathrm{~m} .1 \mathrm{H})$, 3.29-3.26 (m, 1H), 3.15* (dd, 1H, $J=13.6,5.6 \mathrm{~Hz}), 3.03-2.97$ (m, 1H), 2.90* (dd, 1H, $J=13.6$, $10.0 \mathrm{~Hz}), 2.74(\mathrm{dd}, 1 \mathrm{H}, J=14.3,10.1 \mathrm{~Hz}) ;{ }^{13} \mathrm{C} \mathrm{NMR}\left(100 \mathrm{MHz}, \mathrm{CDCl}_{3}\right) \delta 170.8,168.4,146.5$, 145.3, 143.7, 138.0, 131.6, 129.6, 129.4, 128.9, 128.8, 127.4, 127.1, 111.8, 109.0, 108.9, 96.4, 94.0, 53.6, 52.9, 52.8, 43.0, 42.6, 40.8, 40.2, 35.5, 33.4; IR (thin film) v 3063, 3029, 2953, 1781, 1734, 1495, 1455, 1264, 1225, 1201, 1174, 1085, $1009 \mathrm{~cm}^{-1}$; HRMS (ESI) calcd for $\mathrm{C}_{18} \mathrm{H}_{16} \mathrm{O}_{5}$ $[\mathrm{M}+\mathrm{Na}]^{+} 335.0889$, found $335.0890 ; 99 \%$ ee $(3 S, 4 S)$-isomer as determined by HPLC (AD-H, $20: 1$ hexanes/i-PrOH $), t_{\mathrm{r}}(3 S, 4 S)=31.9 \mathrm{~min}, t_{\mathrm{r}}(3 R, 4 R)=22.6 \mathrm{~min}$.
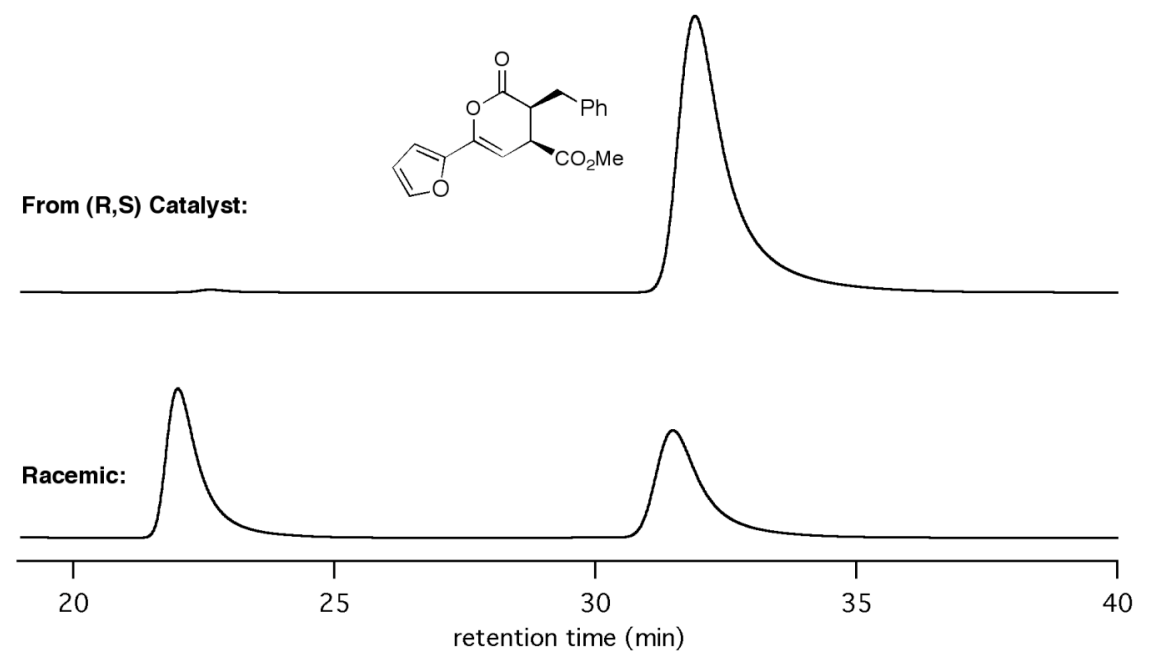


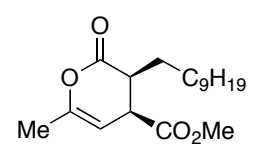

(3S,4S)-Methyl 3-decyl-6-methyl-2-oxo-3,4-dihydro-2H-pyran-4-carboxylate (Table 1, entry

7). Prepared according to the general procedure from 2-chlorododecanal and (E)-methyl 4oxopent-2-enoate using $0.5 \mathrm{~mol} \% \mathbf{1}$ as the catalyst in $71 \%$ yield as a single diastereomer (white solid). $[\alpha]_{\mathrm{D}}{ }^{20}\left(\mathrm{c} 1.20, \mathrm{CHCl}_{3}\right)=+204.8 ; \mathrm{mp}=40-42{ }^{\circ} \mathrm{C} ;{ }^{1} \mathrm{H} \mathrm{NMR}\left(400 \mathrm{MHz}, \mathrm{CDCl}_{3}\right) \delta 5.09$ (dd, $1 \mathrm{H}, J=6.3,1.1 \mathrm{~Hz}), 3.68(\mathrm{~s}, 3 \mathrm{H}), 3.30(\mathrm{dt}, 1 \mathrm{H}, J=6.3,1.0 \mathrm{~Hz}), 2.66(\mathrm{dd}, 1 \mathrm{H}, J=7.0,6.3 \mathrm{~Hz})$, $1.90(\mathrm{t}, 3 \mathrm{H}, J=1.0 \mathrm{~Hz}), 1.35-1.24(\mathrm{~m}, 18 \mathrm{H}), 0.86(\mathrm{t}, 3 \mathrm{H}, J=6.8 \mathrm{~Hz}) ;{ }^{13} \mathrm{C} \mathrm{NMR}(100 \mathrm{MHz}$, $\left.\mathrm{CDCl}_{3}\right) \delta 171.4,169.8,152.3,98.4,52.5,41.5,40.6,32.0,29.7,29.7,29.5,29.5,27.3,27.2,22.8$, 18.9, 14.3; IR (thin film) v 3062, 2925, 2854, 1777, 1740, 1463, 1436, 1383, 1167, $1142 \mathrm{~cm}^{-1}$; HRMS (ESI) calcd for $\mathrm{C}_{18} \mathrm{H}_{30} \mathrm{O}_{4}[\mathrm{M}+\mathrm{Na}]^{+} 333.1996$, found 333.2021; >99\% ee $(3 S$, $4 S$ )-isomer as determined by HPLC (OD-H, 20:1 hexanes/i-PrOH), $t_{\mathrm{r}}(3 S, 4 S)=6.7 \min , t_{\mathrm{r}}(3 R, 4 R)=6.0$ $\min$.
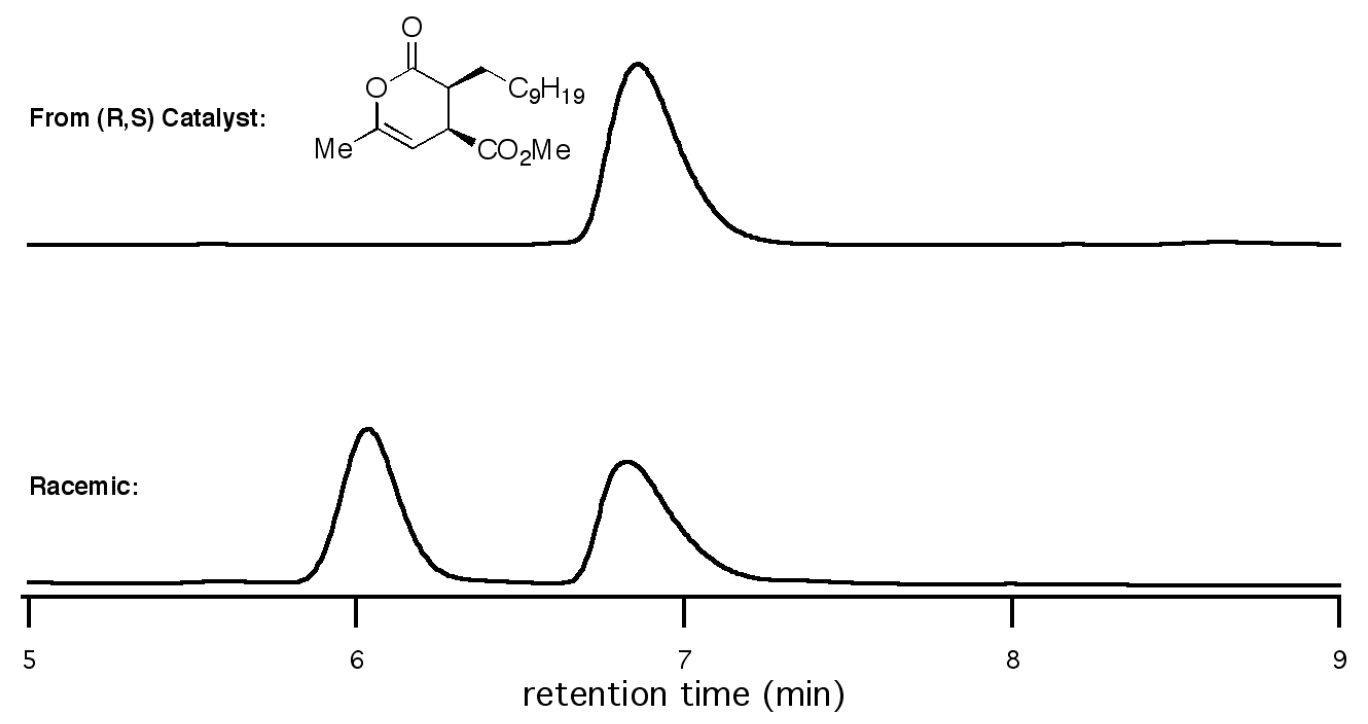


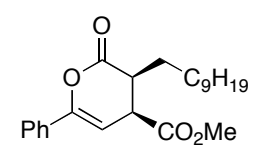

(3S,4S)-Methyl 3-decyl-6-phenyl-2-oxo-3,4-dihydro-2H-pyran-4-carboxylate (Table 1, entry

8). Prepared according to the general procedure from 2-chlorododecanal and (E)-methyl 4-oxo4-phenylbut-2-enoate using $0.5 \mathrm{~mol} \% \mathbf{1}$ as the catalyst in $90 \%$ yield as a single diastereomer (white solid). $[\alpha]_{\mathrm{D}}{ }^{20}\left(\mathrm{c} 1.15, \mathrm{CHCl}_{3}\right)=+154.3 ; \mathrm{mp}=108-111{ }^{\circ} \mathrm{C} ;{ }^{1} \mathrm{H} \mathrm{NMR}\left(400 \mathrm{MHz}, \mathrm{CDCl}_{3}\right) \delta$ 7.66-7.63 (m, 2H), 7.40-7.37 (m, 3H), $5.89(\mathrm{~d}, 1 \mathrm{H}, J=6.4 \mathrm{~Hz}), 3.73(\mathrm{~s}, 3 \mathrm{H}), 3.57(\mathrm{t}, 1 \mathrm{H}, J=6.3$ $\mathrm{Hz}), 2.76(\mathrm{q}, 1 \mathrm{H}, J=6.3 \mathrm{~Hz}), 2.04(\mathrm{t}, 1 \mathrm{H}, J=7.0 \mathrm{~Hz}), 1.47-1.26(\mathrm{~m}, 18 \mathrm{H}), 0.88$ (t, 3H, $J=6.8$ $\mathrm{Hz}) ;{ }^{13} \mathrm{C} \mathrm{NMR}\left(100 \mathrm{MHz}, \mathrm{CDCl}_{3}\right) \delta$ 171.0, 169.5, 152.4, 132.0, 129.8, 128.8, 125.0, 97.9, 52.7, 41.9, 40.7, 32.1, 29.8, 29.8, 29.6, 29.6, 27.4, 27.3, 22.9, 14.4; IR (thin film) v 3062, 2916, 2846, 1758, 1722, 1464, 1339, 1176, $1115 \mathrm{~cm}^{-1}$; HRMS (ESI) calcd for $\mathrm{C}_{23} \mathrm{H}_{32} \mathrm{O}_{4}[\mathrm{M}+\mathrm{Na}]^{+} 395.2192$, found 395.2192; $99 \%$ ee $(3 S, 4 S)$-isomer as determined by HPLC (AD-H, 9:1 hexanes/i-PrOH), $t_{\mathrm{r}}(3 S, 4 S)=7.2 \mathrm{~min}, t_{\mathrm{r}}(3 R, 4 R)=10.3 \mathrm{~min}$.
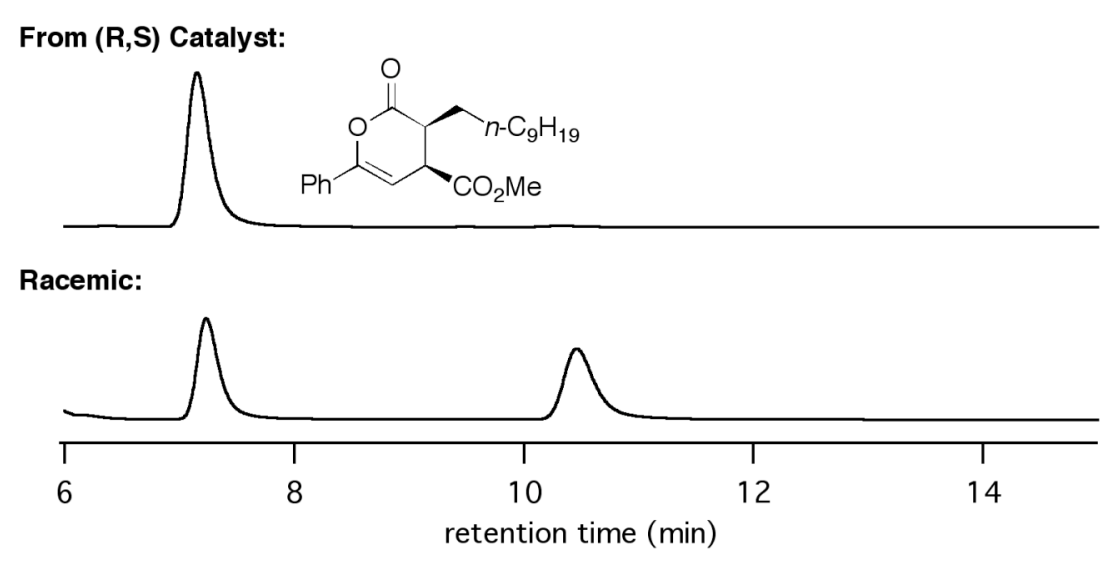

(3R,4S)-Methyl 3-((tert-butyldimethylsilyloxy)methyl)-6-phenyl-2-oxo-3,4-dihydro-2Hpyran-4-carboxylate (Table 1, entry 9). Prepared according to the general procedure from 3(tert-butyldimethylsilyloxy)-2-chloropropanal and (E)-methyl 4-oxo-4-phenylbut-2-enoate using 
$0.5 \mathrm{~mol} \% \mathbf{1}$ as the catalyst in $80 \%$ yield as a 3:1 mixture of diastereomers. The diastereomers were separated by silica gel chromatography (15:1 hexanes/EtOAc) and characterized separately. $[\alpha]_{\mathrm{D}}{ }^{20}\left(\mathrm{c} 1.07, \mathrm{CHCl}_{3}\right)=+133.6 ;{ }^{1} \mathrm{H}$ NMR $\left(400 \mathrm{MHz}, \mathrm{CDCl}_{3}\right) \delta$ 7.65-7.62 (m, 2H), 7.40-7.37 $(\mathrm{m}, 3 \mathrm{H}), 5.94(\mathrm{~d}, 1 \mathrm{H}, J=7.1 \mathrm{~Hz}), 4.26(\mathrm{dd}, 1 \mathrm{H}, J=10.6,5.7 \mathrm{~Hz}), 3.88(\mathrm{t}, 1 \mathrm{H}, J=10.1 \mathrm{~Hz})$, 3.76(dd, 1H, $J=13.7,7.4 \mathrm{~Hz}), 3.72(\mathrm{~s}, 3 \mathrm{H}), 3.08-3.02(\mathrm{~m}, 1 \mathrm{H}), 0.89$ (s, 9H), 0.07 (s, 3H), 0.06 $(\mathrm{s}, 3 \mathrm{H}) ;{ }^{13} \mathrm{C} \mathrm{NMR}\left(100 \mathrm{MHz}, \mathrm{CDCl}_{3}\right) \delta 170.9,167.7,152.5,131.9,129.8,128.8,125.0,98.1$, 59.7, 52.6, 42.5, 38.7, 25.9, 18.4, -5.4, -5.6; IR (thin film) v 3062, 2953, 2929, 2886, 2857, 1778, 1739, 1254, 1197, $1176 \mathrm{~cm}^{-1}$; HRMS (EI) calcd for $\mathrm{C}_{20} \mathrm{H}_{25} \mathrm{O}_{5} \mathrm{Si}[\mathrm{M}]^{+}$376.1706, found 376.1697; $97 \%$ ee $(3 R, 4 S)$-isomer as determined by HPLC (AD-H, 100:1 hexanes/i-PrOH), $t_{\mathrm{r}}(3 R, 4 S)=21.8 \mathrm{~min}, t_{\mathrm{r}}(3 S, 4 R)=15.2 \mathrm{~min}$.

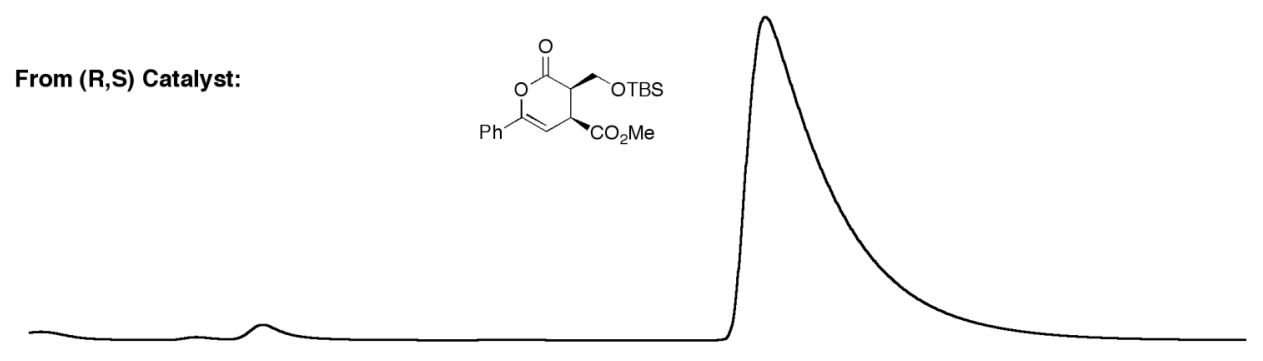

Racemic:
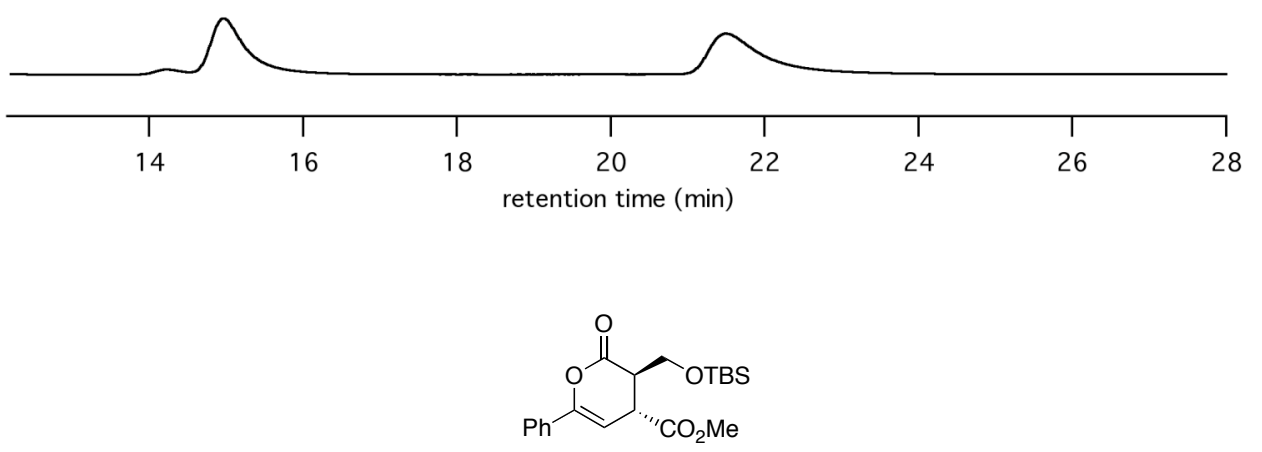

(3R,4R)-Methyl 3-((tert-butyldimethylsilyloxy)methyl)-6-phenyl-2-oxo-3,4-dihydro-2Hpyran-4-carboxylate ${ }^{1} \mathrm{H}$ NMR $\left(400 \mathrm{MHz} \mathrm{CDCl}_{3}\right) \delta 7.64-7.61(\mathrm{~m}, 2 \mathrm{H}), 7.40-7.37(\mathrm{~m}, 3 \mathrm{H})$, $5.75(\mathrm{~d}, 1 \mathrm{H}, J=7.1 \mathrm{~Hz}), 4.05(\mathrm{dd}, 1 \mathrm{H}, J=10.1,6.3 \mathrm{~Hz}), 3.89(\mathrm{dd}, 1 \mathrm{H}, J=10.1,4.2 \mathrm{~Hz}), 3.80-$ $3.77(\mathrm{~m}, 1 \mathrm{H}), 3.78(\mathrm{~s}, 3 \mathrm{H}), 3.30-3.25(\mathrm{~m}, 1 \mathrm{H}), 0.87(\mathrm{~s}, 9 \mathrm{H}), 0.052(\mathrm{~s}, 3 \mathrm{H}), 0.048(\mathrm{~s}, 3 \mathrm{H}) ;{ }^{13} \mathrm{C}$ 
NMR (100 MHz, $\left.\mathrm{CDCl}_{3}\right) \delta 172.2,167.6,150.5,131.9,129.7,128.7,125.0,97.2,61.0,52.9$, 43.2, 39.5, 26.0, 18.5, -5.4; IR (thin film) v 3062, 2953, 2929, 2886, 2857, 1778, 1739, 1254, 1197, $1176 \mathrm{~cm}^{-1}$; HRMS (EI) calcd for $\mathrm{C}_{20} \mathrm{H}_{25} \mathrm{O}_{5} \mathrm{Si}[\mathrm{M}]^{+} 376.1706$, found 376.1697; $97 \%$ ee (3R, $4 R)$-isomer as determined by HPLC (AD-H, 100:1 hexanes/i-PrOH), $t_{\mathrm{r}}(3 R, 4 R)=24.6 \mathrm{~min}, t_{\mathrm{r}}(3 S$, $4 S)=17.1 \mathrm{~min}$.
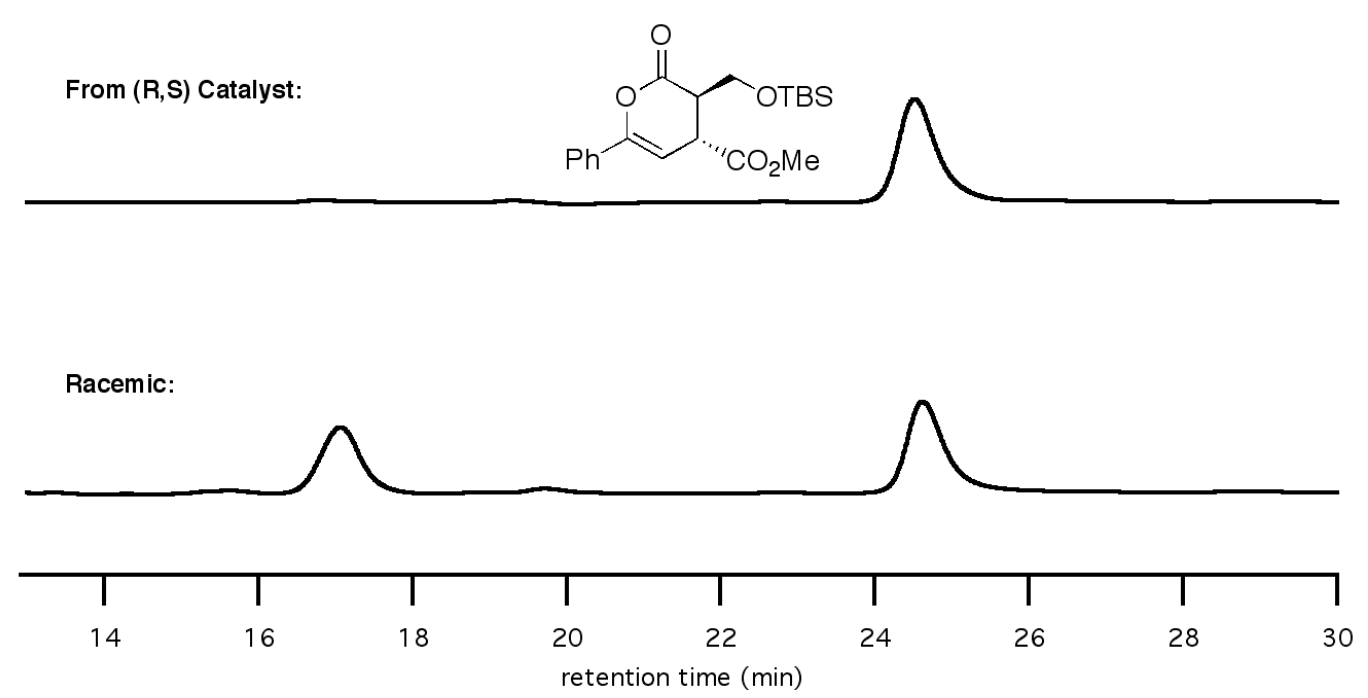


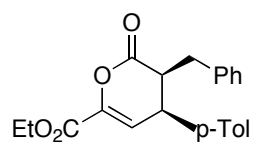

(4S,5S)-Ethyl 5-benzyl-6-oxo-4-para-tolyl-5,6-dihydro-4H-pyran-2-carboxylate (Table 2, entry 1). Prepared according to the general procedure from 2-chloro-hydrocinnamaldehyde and (E)-ethyl 2-oxo-4-p-tolylbut-3-enoate using $2 \mathrm{~mol} \% \mathbf{1}$ as the catalyst in $74 \%$ yield as a single diastereomer (colorless oil). $[\alpha]_{\mathrm{D}}{ }^{20}\left(\mathrm{c} 1.02, \mathrm{CHCl}_{3}\right)=+310.3 ;{ }^{1} \mathrm{H} \mathrm{NMR}\left(400 \mathrm{MHz}, \mathrm{CDCl}_{3}\right) \delta$ 7.32-7.24 (m, 3H), 7.16-7.07 (m, 4H), 6.93-6.91 (m, 2H), 6.65 (d, 1H, J = 6.8 Hz), 4.30 (q, 1H, $J=7.1 \mathrm{~Hz}), 3.58(\mathrm{t}, 1 \mathrm{H}, \mathrm{J}=6.7 \mathrm{~Hz}), 3.29-3.23(\mathrm{~m}, 2 \mathrm{H}), 2.41(\mathrm{dd}, 1 \mathrm{H}, J=15.8,10.9 \mathrm{~Hz}), 2.35(\mathrm{~s}$, $3 \mathrm{H}), 1.33(\mathrm{t}, 3 \mathrm{H}, J=7.1 \mathrm{~Hz}) ;{ }^{13} \mathrm{C} \mathrm{NMR}\left(100 \mathrm{MHz}, \mathrm{CDCl}_{3}\right) \delta 168.9,160.7,142.1,138.3,132.8$, 130.0, 129.1, 128.8, 128.4, 126.9, 118.9, 62.1, 45.0, 40.3, 32.1, 21.3, 14.3; IR (thin film) v 3062, $3027,2982,2925,1776,1732,1316,1267,1106 \mathrm{~cm}^{-1}$; HRMS (ESI) calcd for $\mathrm{C}_{22} \mathrm{H}_{22} \mathrm{O}_{4}[\mathrm{M}+\mathrm{Na}]^{+}$ 373.1410, found 373.1409; $97 \%$ ee $(4 S, 5 S)$-isomer as determined by HPLC (AD-H, 9:1 hexanes/i-PrOH $), t_{\mathrm{r}}(4 S, 5 S)=21.7 \mathrm{~min}, t_{\mathrm{r}}(4 R, 5 R)=13.4 \mathrm{~min}$.

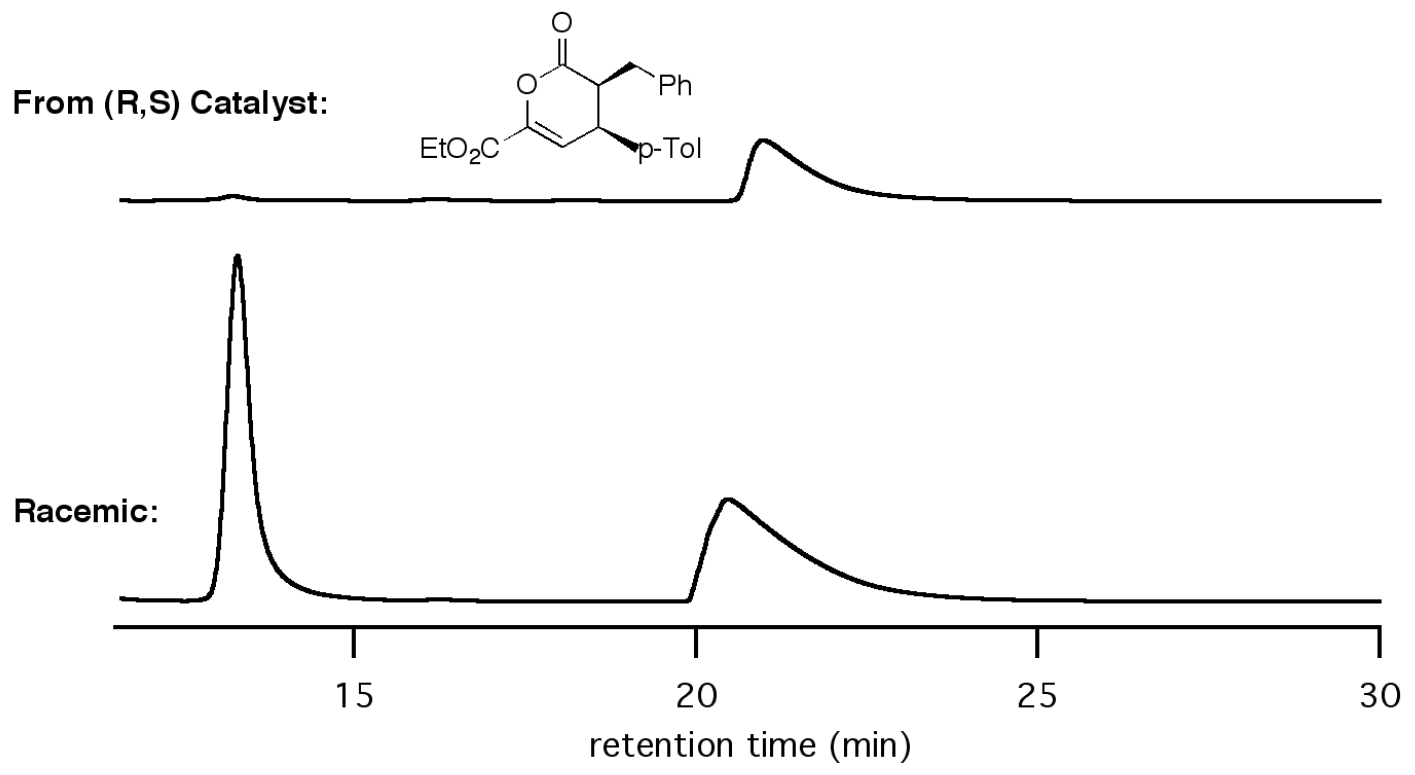




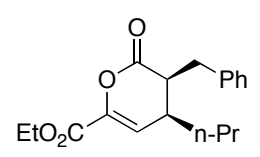

(4S,5S)-Ethyl 5-benzyl-6-oxo-4-propyl-5,6-dihydro-4H-pyran-2-carboxylate (Table 2, entry

2). Prepared according to the general procedure from 2-chloro-hydrocinnamaldehyde and $(E)$ ethyl 2-oxohept-3-enoate using $2 \mathrm{~mol} \% \mathbf{1}$ as the catalyst in $84 \%$ yield as a single diastereomer (white solid). $[\alpha]_{\mathrm{D}}{ }^{20}\left(\mathrm{c} 1.01, \mathrm{CHCl}_{3}\right)=+115.3 ; \mathrm{mp}=77-81{ }^{\circ} \mathrm{C} ;{ }^{1} \mathrm{H}$ NMR $\left(400 \mathrm{MHz}, \mathrm{CDCl}_{3}\right) \delta$ $7.32-7.17(\mathrm{~m}, 5 \mathrm{H}), 6.57(\mathrm{~d}, 1 \mathrm{H}, J=6.5 \mathrm{~Hz}), 4.28(\mathrm{dq}, 2 \mathrm{H}, J=7.2,1.5 \mathrm{~Hz}), 3.33(\mathrm{dd}, 1 \mathrm{H}, \mathrm{J}=$ 14.3, 5.5 Hz), 2.95-2.90 (m, 1H), $2.74(\mathrm{dd}, 1 \mathrm{H}, J=14.3,9.5 \mathrm{~Hz}), 2.42-2.39$ (m, 1H), 1.62-1.58 $(\mathrm{m}, 2 \mathrm{H}), 1.42-1.20(\mathrm{~m}, 5 \mathrm{H}), 0.88(\mathrm{t}, 3 \mathrm{H}, J=7.1 \mathrm{~Hz}) ;{ }^{13} \mathrm{C} \mathrm{NMR}\left(100 \mathrm{MHz}, \mathrm{CDCl}_{3}\right) \delta 169.7$, $160.7,142.2$, 138.4, 128.9, 126.9, 120.5, 62.0, 44.4, 33.2, 32.0, 31.1, 20.0, 14.4, 14.3; IR (thin film) $v$ 3062, 3027, 2959, 2924, 1776, 1736, 1455, 1370, 1262, $1109 \mathrm{~cm}^{-1}$; HRMS (ESI) calcd for $\mathrm{C}_{18} \mathrm{H}_{22} \mathrm{O}_{4}[\mathrm{M}+\mathrm{Na}]^{+}$325.1410, found 325.1398; 98\% ee $(4 S, 5 S)$-isomer as determined by HPLC (AS-H, 9:1 hexanes/i-PrOH $), t_{\mathrm{r}}(4 S, 5 S)=18.9 \mathrm{~min}, t_{\mathrm{r}}(4 R, 5 R)=11.9 \mathrm{~min}$.

From $(\mathbf{R}, \mathbf{S})$ Catalyst:
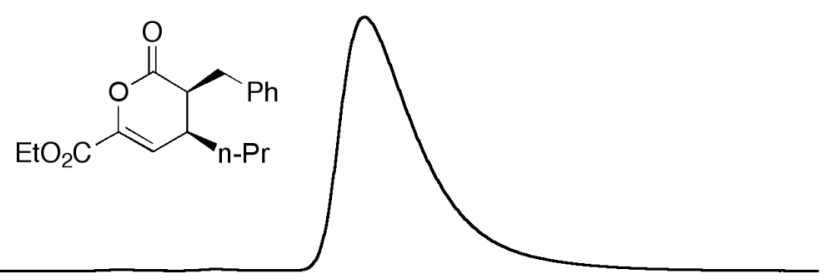

Racemic:

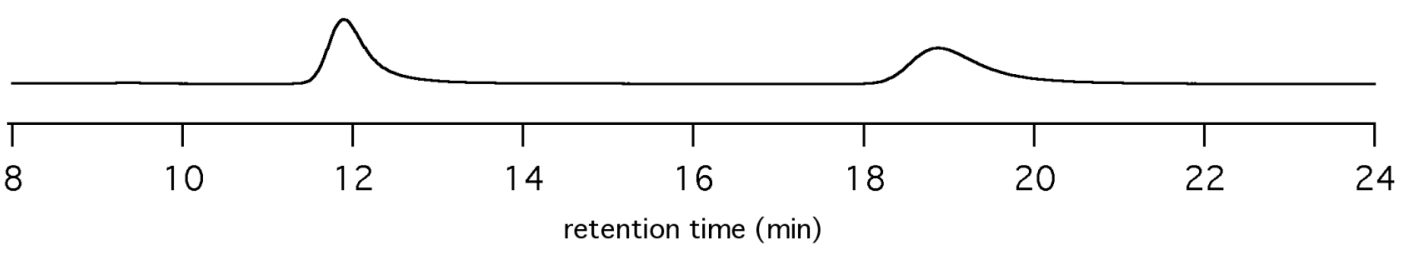




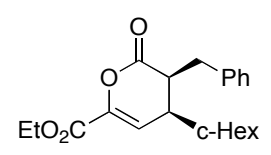

(4S,5S)-Ethyl 5-benzyl-6-oxo-4-cyclohexyl-5,6-dihydro-4H-pyran-2-carboxylate (Table 2, entry 3). Prepared according to the general procedure from 2-chloro-hydrocinnamaldehyde and (E)-ethyl 4-cyclohexyl-2-oxobut-3-enoate using $2 \mathrm{~mol} \% \mathbf{1}$ as the catalyst in $85 \%$ yield as a single diastereomer (colorless oil). $[\alpha]_{\mathrm{D}}{ }^{20}\left(\mathrm{c} 1.20, \mathrm{CHCl}_{3}\right)=+83.5 ;{ }^{1} \mathrm{H} \mathrm{NMR}\left(400 \mathrm{MHz}, \mathrm{CDCl}_{3}\right) \delta$ 7.33-7.21 (m, 5H), $6.48(\mathrm{~d}, 1 \mathrm{H}, J=6.5 \mathrm{~Hz}), 4.30(\mathrm{dq}, 1 \mathrm{H}, J=7.2,1.8 \mathrm{~Hz}), 3.38(\mathrm{dd}, 1 \mathrm{H}, \mathrm{J}=$ 14.3, 5.7 Hz), 2.97-2.91 (m, 1H), $2.77(\mathrm{dd}, 1 \mathrm{H}, J=14.3,9.1 \mathrm{~Hz}), 2.45-2.41(\mathrm{~m}, 1 \mathrm{H}), 1.76-1.62$ $(\mathrm{m}, 5 \mathrm{H}), 1.47-1.44(\mathrm{~m}, 1 \mathrm{H}), 1.34(\mathrm{t}, 3 \mathrm{H}, J=7.2 \mathrm{~Hz}), 1.27-1.06(\mathrm{~m}, 4 \mathrm{H}), 0.93-0.89(\mathrm{~m}, 1 \mathrm{H}) ;{ }^{13} \mathrm{C}$ NMR (100 MHz, $\left.\mathrm{CDCl}_{3}\right) \delta 170.4,160.7,142.8,138.6,128.9,126.9,117.6,62.0,43.0,39.4$, 37.7, 31.7, 31.4, 27.2, 26.8, 26.1, 14.3; IR (thin film) v 3520, 3086, 3028, 2981, 2928, 2853, 1770, 1731, 1660, 1453, 1369, 1309, 1262, 1120, 1094, $1013 \mathrm{~cm}^{-1}$; HRMS (ESI) calcd for $\mathrm{C}_{21} \mathrm{H}_{26} \mathrm{O}_{4}[\mathrm{M}+\mathrm{Na}]^{+} 365.1723$, found $365.1721 ; 95 \%$ ee $(4 S, 5 S)$-isomer as determined by HPLC $(\mathrm{AS}-\mathrm{H}, 20: 1$ hexanes/i-PrOH$), t_{\mathrm{r}}(4 S, 5 S)=19.1 \mathrm{~min}, t_{\mathrm{r}}(4 R, 5 R)=15.7 \mathrm{~min}$.

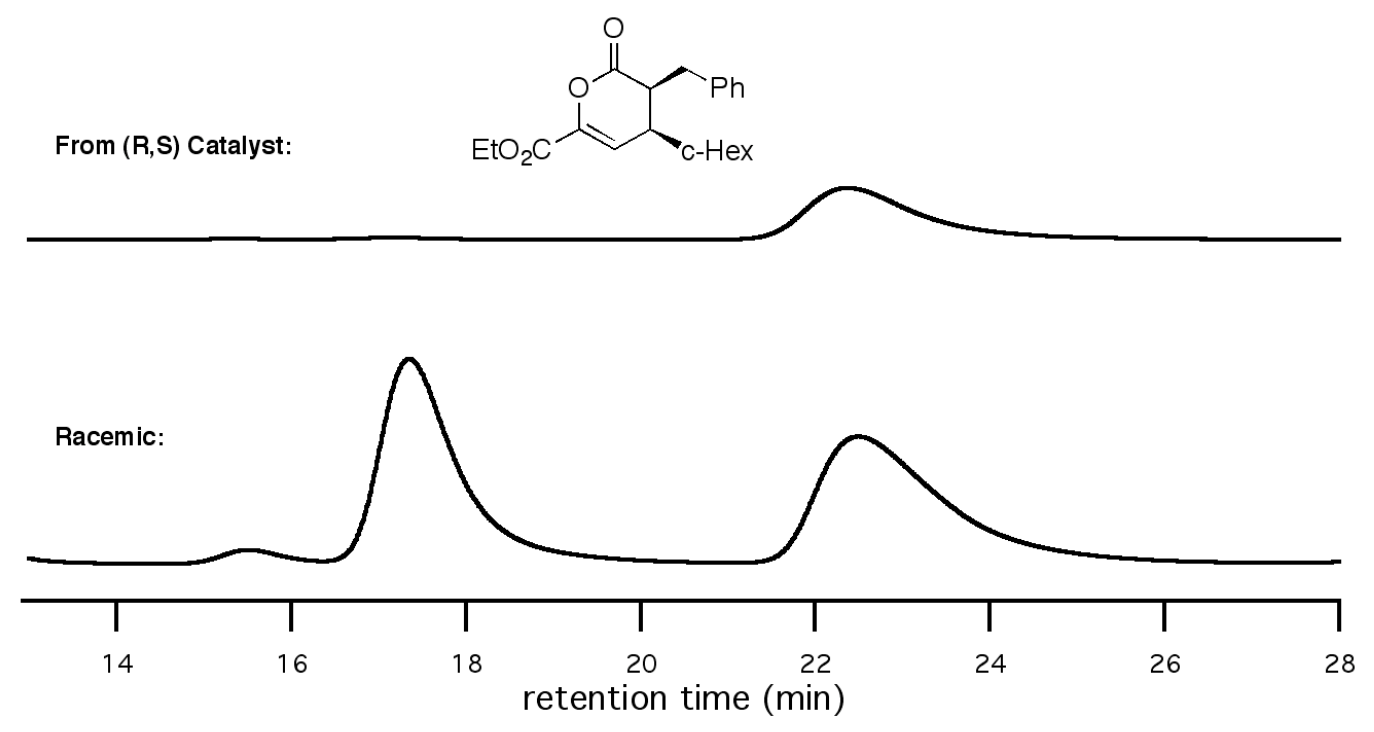


<smiles>CCCC1C(=O)OC(C(=O)OCC)=CC1[Pb]</smiles>

(4S,5S)-Ethyl 5-decyl-6-oxo-4-para-tolyl-5,6-dihydro-4H-pyran-2-carboxylate (Table 2, entry

4 ). Prepared according to the general procedure from 2-chlorododecanal and (E)-ethyl 2-oxo-4p-tolylbut-3-enoate using $2 \mathrm{~mol} \% \mathbf{1}$ as the catalyst in $70 \%$ yield as a single diastereomer (colorless oil). $[\alpha]_{\mathrm{D}}{ }^{20}\left(\mathrm{c} 1.31, \mathrm{CHCl}_{3}\right)=+154.3 ;{ }^{1} \mathrm{H}$ NMR $\left(400 \mathrm{MHz}, \mathrm{CDCl}_{3}\right) \delta 7.32-7.24(\mathrm{~m}$, $3 \mathrm{H}), 7.12(\mathrm{~d}, 2 \mathrm{H}, J=8.1 \mathrm{~Hz}), 6.99(\mathrm{~d}, 2 \mathrm{H}, J=8.1 \mathrm{hz}), 6.70(\mathrm{~d}, 1 \mathrm{H}, J=6.5 \mathrm{~Hz}), 4.32(\mathrm{q}, 1 \mathrm{H}, J=$ $7.2 \mathrm{~Hz}), 3.77$ (t, 1H, J = 6.8 Hz), 2.81 (q, 1H, J=6.8 Hz), 2.33 (s, 3H), 1.72-1.63 (m, 2H), 1.37$1.14(\mathrm{~m}, 19 \mathrm{H}), 0.88(\mathrm{t}, 3 \mathrm{H}, J=6.8 \mathrm{~Hz}) ;{ }^{13} \mathrm{C} \mathrm{NMR}\left(100 \mathrm{MHz}, \mathrm{CDCl}_{3}\right) \delta 169.2,160.8,142.3$, 138.1, 133.1, 130.0, 128.1, 118.6, 62.1, 43.6, 41.2, 32.1, 29.7, 29.7, 29.5, 27.3, 26.4, 22.9, 21.3, 14.4, 14.3; IR (thin film) v 3027, 2925, 2854, 1777, 1739, 1659, 1464, 1370, 1316, 1260, 1104 $\mathrm{cm}^{-1}$; HRMS (EI) calcd for $\mathrm{C}_{25} \mathrm{H}_{36} \mathrm{O}_{4}[\mathrm{M}]^{+} 400.2614$, found 400.2613; $99 \%$ ee $(4 S, 5 S)$-isomer as determined by HPLC (OD-H, 20:1 hexanes/i-PrOH), $t_{\mathrm{r}}(4 S, 5 S)=8.0 \mathrm{~min}, t_{\mathrm{r}}(4 R, 5 R)=9.2 \mathrm{~min}$.
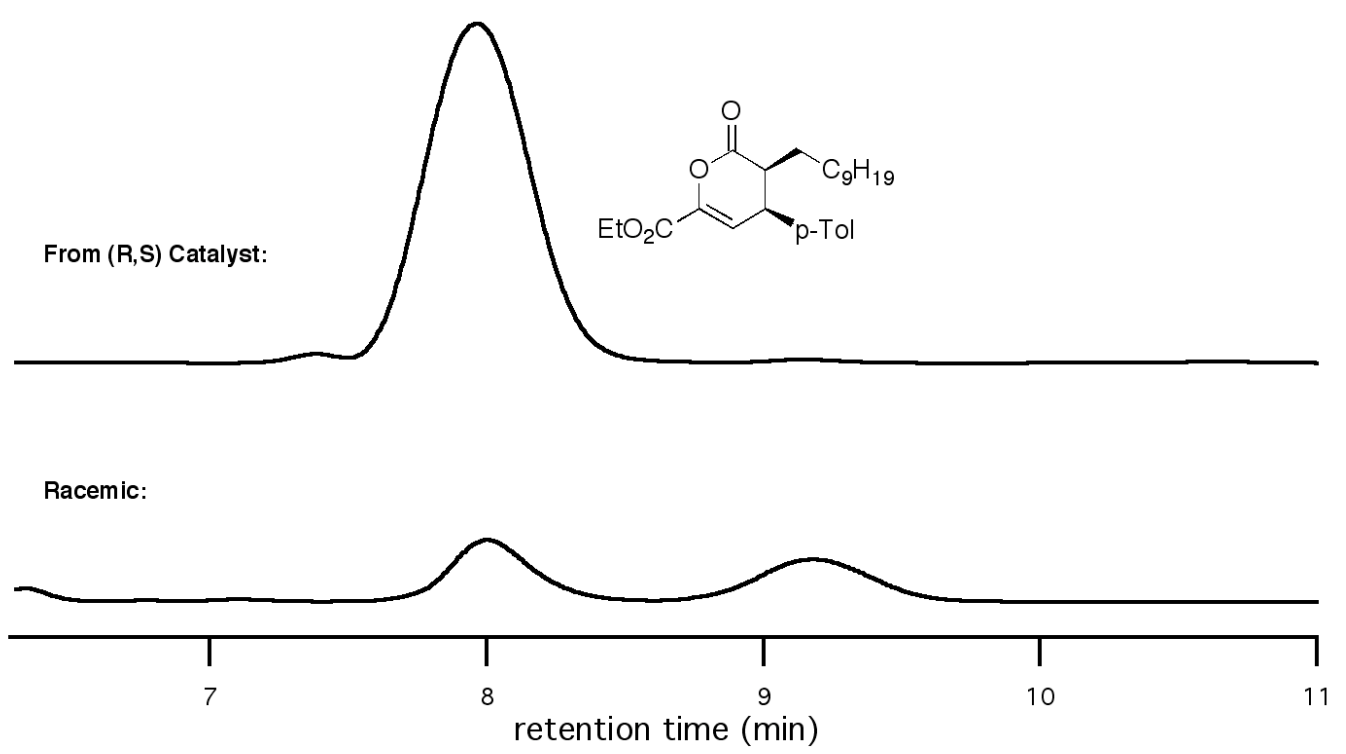
<smiles>CCOC(=O)C1=CC([Pb])C(COC)C(=O)O1</smiles>

(4S,5R)-Ethyl 5-((tert-butyldimethylsilyloxy)methyl)-6-oxo-4-para-tolyl-5,6-dihydro-4Hpyran-2-carboxylate (Table 2, entry 5). Prepared according to the general procedure from 3(tert-butyldimethylsilyloxy)-2-chloropropanal and (E)-ethyl 2-oxo-4-p-tolylbut-3-enoate using 2 mol $\% 1$ as the catalyst in $83 \%$ yield as a single diastereomer (white solid). $[\alpha]_{\mathrm{D}}{ }^{20}\left(\mathrm{c} 1.27, \mathrm{CHCl}_{3}\right.$ ) $=+244.6 ; \mathrm{mp}=60-61{ }^{\circ} \mathrm{C} ;{ }^{1} \mathrm{H}$ NMR $\left(400 \mathrm{MHz}, \mathrm{CDCl}_{3}\right) \delta 7.32-7.24(\mathrm{~m}, 3 \mathrm{H}), 7.12(\mathrm{~d}, 2 \mathrm{H}, J=8.1$ $\mathrm{Hz}), 6.99(\mathrm{~d}, 2 \mathrm{H}, J=8.1 \mathrm{hz}), 6.70(\mathrm{~d}, 1 \mathrm{H}, J=6.5 \mathrm{~Hz}), 4.32(\mathrm{q}, 1 \mathrm{H}, J=7.2 \mathrm{~Hz}), 3.77(\mathrm{t}, 1 \mathrm{H}, \mathrm{J}=$ $6.8 \mathrm{~Hz}), 2.81(\mathrm{q}, 1 \mathrm{H}, J=6.8 \mathrm{~Hz}), 2.33(\mathrm{~s}, 3 \mathrm{H}), 1.72-1.63(\mathrm{~m}, 2 \mathrm{H}), 1.37-1.14(\mathrm{~m}, 19 \mathrm{H}), 0.88(\mathrm{t}$, $3 \mathrm{H}, J=6.8 \mathrm{~Hz}) ;{ }^{13} \mathrm{C} \mathrm{NMR}\left(100 \mathrm{MHz}, \mathrm{CDCl}_{3}\right) \delta 167.3,160.7,142.6,138.2,132.0,129.8,128.5$, $118.9,62.1,58.7,45.8,38.3,26.0,21.3,18.3,14.4,-5.3,-5.4$; IR (thin film) v 3027, 2953, 2929, 2857, 1776, 1740, 1658, 1471, 1370, 1317, 1262, $1084 \mathrm{~cm}^{-1}$; HRMS (EI) calcd for $\mathrm{C}_{25} \mathrm{H}_{36} \mathrm{O}_{4}$ [M$\mathrm{Bu}]^{+} 347.1315$, found $347.1305 ; 95 \%$ ee $(4 S, 5 R)$-isomer as determined by HPLC (AD-H, 100:1 hexanes/i-PrOH $), t_{\mathrm{r}}(4 S, 5 R)=8.5 \mathrm{~min}, t_{\mathrm{r}}(4 R, 5 S)=10.0 \mathrm{~min}$.

From $(R, S)$ Catalyst:
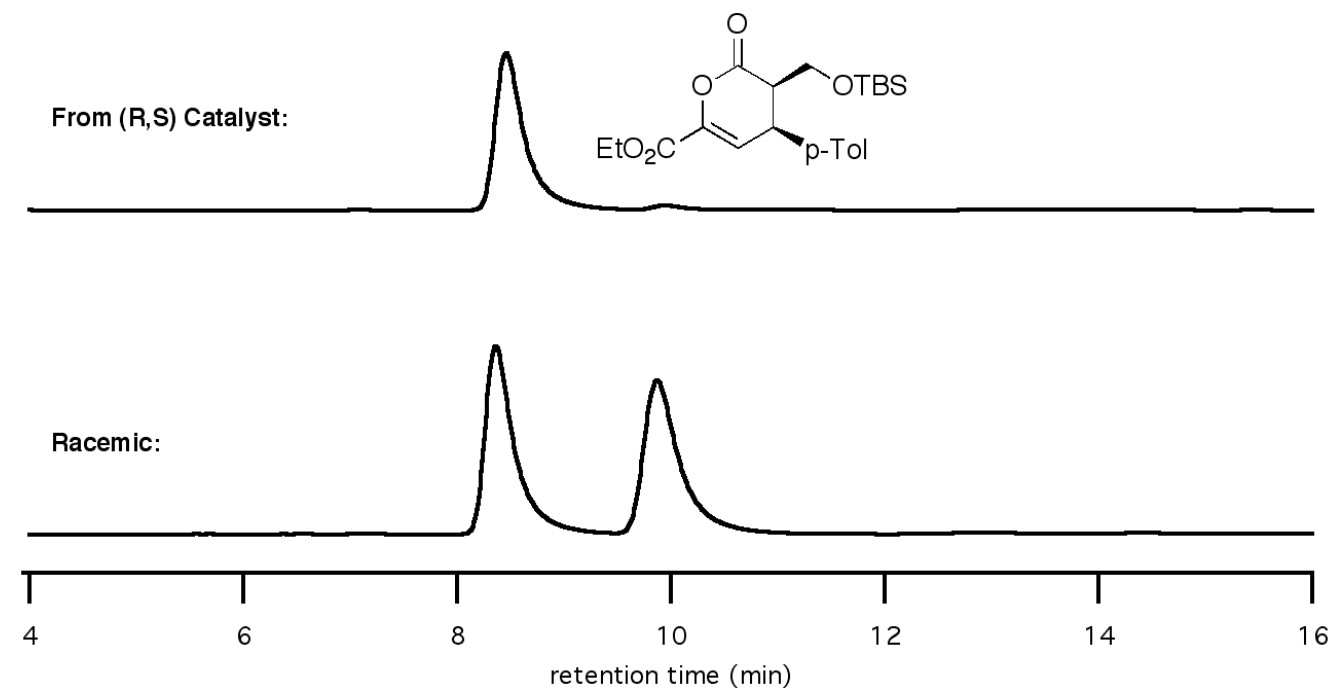


\section{Preparation of starting materials.}

All the racemic and chiral 2-chloroaldehydes were prepared according to the previous literature procedures. $^{2,3}$ The substituted 4-oxo-enoates (Table 1, entry 1 9) were prepared as previously reported via one-pot oxidation-stabilized Wittig reactions ${ }^{4}$ to provide the desired enoates.

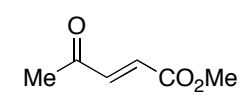

(E)-Methyl 4-oxopent-2-enoate. ${ }^{1} \mathrm{H}$ NMR $\left(400 \mathrm{MHz}, \mathrm{CDCl}_{3}\right) \delta 6.96(\mathrm{~d}, 1 \mathrm{H}, J=16.1 \mathrm{~Hz}), 6.60$ $(\mathrm{d}, 1 \mathrm{H}, J=16.1 \mathrm{hz}), 3.75(\mathrm{~s}, 3 \mathrm{H}), 2.30(\mathrm{~s}, 3 \mathrm{H}) ;{ }^{13} \mathrm{C} \mathrm{NMR}\left(100 \mathrm{MHz}, \mathrm{CDCl}_{3}\right) \delta$ 197.7, 166.0, 140.2, 131.1, 52.5, 28.2; MS (EI) $\mathrm{C}_{6} \mathrm{H}_{8} \mathrm{O}_{3}[\mathrm{M}]^{+} 128$.

The $\beta, \gamma$-unsaturated $\alpha$-ketoesters were prepared by reacting ethyl triphenylphosphoranylidenepyruvate with corresponding aldehydes. $^{5}$

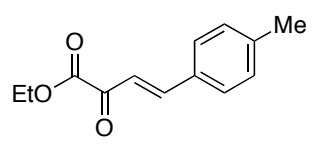

(E)-Ethyl 2-oxo-4-para-tolylbut-3-enoate. ${ }^{1} \mathrm{H} \mathrm{NMR}\left(400 \mathrm{MHz}, \mathrm{CDCl}_{3}\right) \delta 7.85(\mathrm{~d}, 1 \mathrm{H}, J=14.1$ $\mathrm{Hz}), 7.54(\mathrm{~d}, 2 \mathrm{H}, J=8.0 \mathrm{~Hz}), 7.33(\mathrm{~d}, 1 \mathrm{H}, J=16.1 \mathrm{~Hz}), 7.25(\mathrm{~d}, 2 \mathrm{H}, J=8.0 \mathrm{hz}), 4.39(\mathrm{q}, 2 \mathrm{H}, J=$ $7.1 \mathrm{~Hz}), 2.40(\mathrm{~s}, 3 \mathrm{H}), 1.41(\mathrm{t}, 3 \mathrm{H}, J=7.1 \mathrm{~Hz}) ;{ }^{13} \mathrm{C} \mathrm{NMR}\left(100 \mathrm{MHz}, \mathrm{CDCl}_{3}\right) \delta 183.0,162.4$, 148.7, 142.6, 131.4, 130.0, 129.2, 119.6, 62.6, 21.7, 14.2; HRMS (EI) calcd for $\mathrm{C}_{13} \mathrm{H}_{14} \mathrm{O}_{3}[\mathrm{M}]^{+}$ 218.0943, found 218.0951.

(2) Halland, N.; Braunton, A.; Bachmann, S.; Marigo, M.; Jøgensen, K. A. J. Am. Chem. Soc. 2004, 126, 4670-4791.

(3) Brochu, M. P.; Brown, S. P.; MacMillan, D. W. C. J. Am. Chem. Soc. 2004, 126, 4108-4109.

(4) Runcie, K. A.; Taylor, R. J. Chem. Comm. 2002, 974-975.

(5) Meijer, L. H. P.; Pandit, U. K. Tetrahedron, 1985, 41, 467-472. 


\section{Epimerization studies.}

a) Epimerization studies under the catalytic reaction conditions

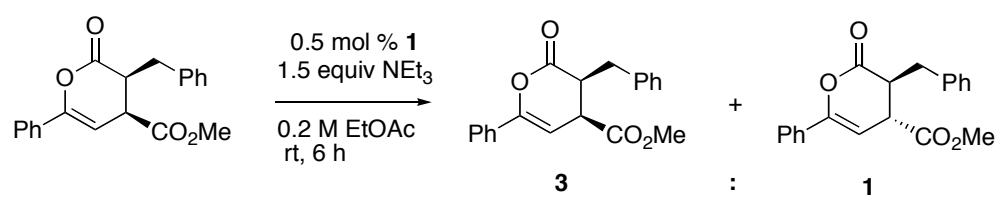

$(3 S, 4 S)$-Methyl 3-benzyl-6-phenyl-2-oxo-3,4-dihydro-2H-pyran-4-carboxylate $(32.2 \mathrm{mg},>20: 1$ dr) in $0.5 \mathrm{ml}$ EtOAc was treated with 1.5 equiv $\mathrm{NEt}_{3}$ and $0.5 \mathrm{~mol} \%$ triazolium salt 1 and the mixture was stirred at $\mathrm{rt}$ for $6 \mathrm{~h}$. The solvent was removed under reduced pressure. ${ }^{1} \mathrm{H}-\mathrm{NMR}$ revealed that the product had epimerized to a 3:1 mixture of diastereomers. The enantiomeric integrity of the products was confirmed by HPLC.

HPLC analysis of major diasteromer (cis): $99 \%$ ee $(3 S, 4 S)$-isomer as determined by HPLC (AD$\mathrm{H}, 9: 1$ hexanes/i-PrOH $), t_{\mathrm{r}}(3 S, 4 S)=32.4 \mathrm{~min}, t_{\mathrm{r}}(3 R, 4 R)=20.3 \mathrm{~min}$.

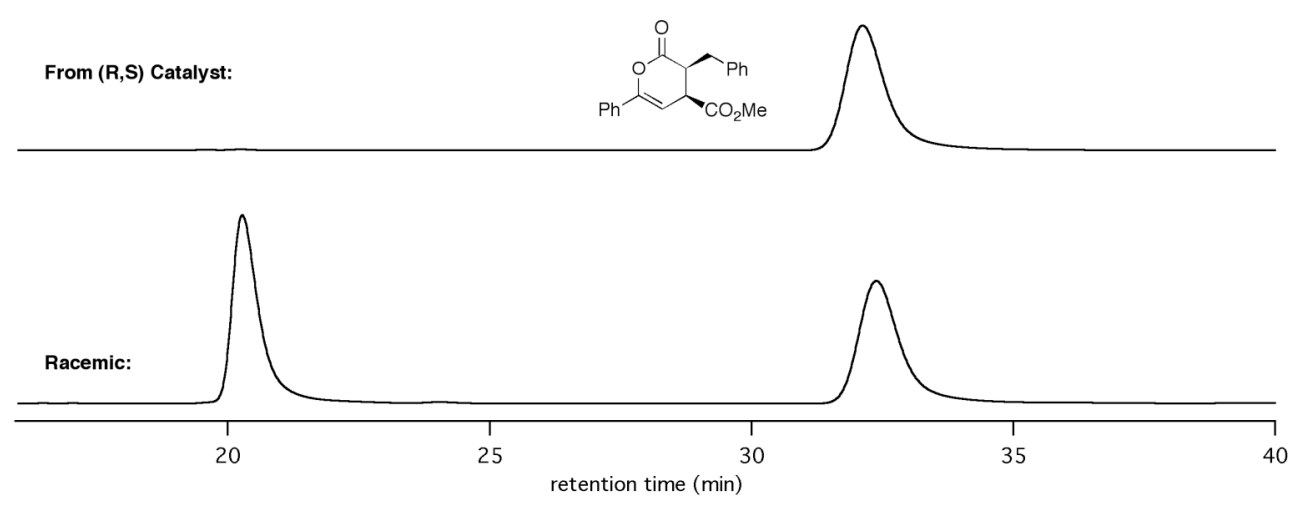

HPLC analysis of major diasteromer (trans): $96 \%$ ee $(3 S, 4 R)$-isomer as determined by HPLC $(\mathrm{AD}-\mathrm{H}, 20: 1$ hexanes/i-PrOH$), t_{\mathrm{r}}(3 S, 4 R)=19.6 \mathrm{~min}, t_{\mathrm{r}}(3 R, 4 S)=21.1 \mathrm{~min}$. 

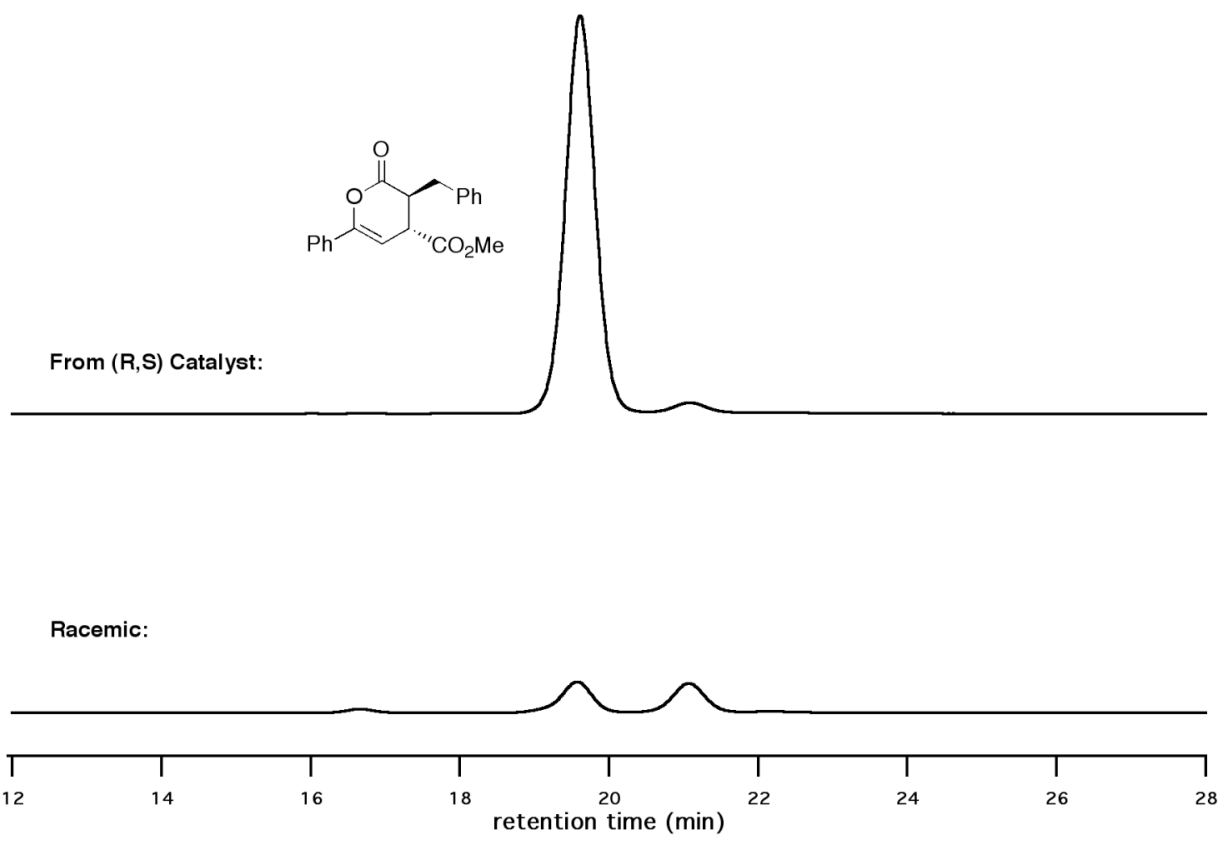

b) Epimerization of a diastereomerically pure dihydropyranone.

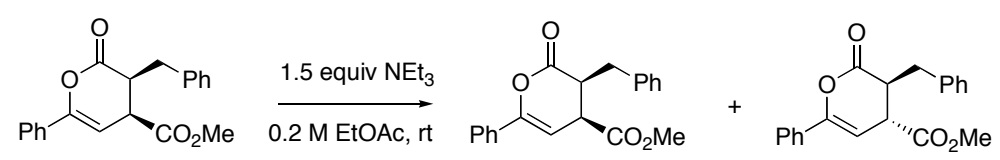

(3S,4S)-Methyl 3-benzyl-6-phenyl-2-oxo-3,4-dihydro-2H-pyran-4-carboxylate (32.2 mg) in 0.5 ml EtOAc was treated with 1.5 equiv $\mathrm{NEt}_{3}$ and the mixture was stirred at rt. The diastereomer ratio of the cis/trans was detected by ${ }^{1} \mathrm{H}-\mathrm{NMR}$, and the results summarized in the table below.

\begin{tabular}{ccccc}
\hline time $(\mathrm{h})$ & 0 & 12 & 24 & 48 \\
\hline $\begin{array}{c}\text { d.r. } \\
(\text { cis/trans })\end{array}$ & $>20: 1$ & $2: 1$ & $1.3: 1$ & $1.2: 1$ \\
\hline
\end{tabular}


Oxadiene Diels-Alder reactions with enantiomerically enriched chloroaldehyde.

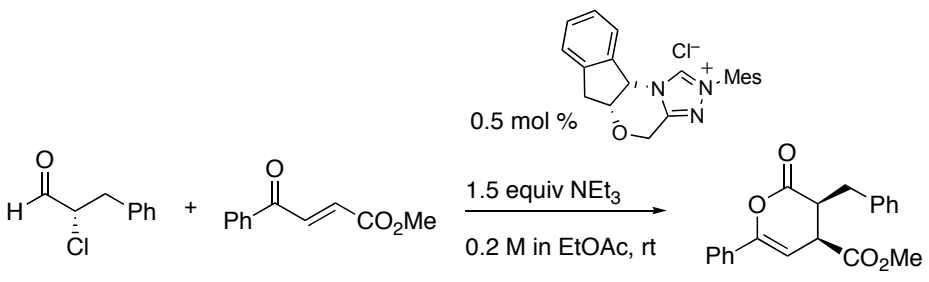

(3S,4S)-methyl 3-benzyl-6-phenyl-2-oxo-3,4-dihydro-2H-pyran-4-carboxylate was prepared according to the general procedure, using enantioenriched 2-chloro-hydrocinnamaldehyde ${ }^{3}$ (97\% ee, $110.2 \mathrm{mg}, 0.65 \mathrm{mmol}, 1.2$ equiv), (E)-methyl 4-oxo-4-phenylbut-2-enoate (103.5 mg, 0.54 mmol, 1.0 equiv), triazolium salt $1(1.0 \mathrm{mg}, 0.5 \mathrm{~mol} \%)$ and $\mathrm{NEt}_{3}(112 \mu \mathrm{L}, 0.82 \mathrm{mmol} 1.5$ equiv). The desired dihydropyranone product was obtained as a white solid (128 mg, 72\% yield, $4.5: 1 \mathrm{dr}, 98 \%$ ee $(3 S, 4 S))$.

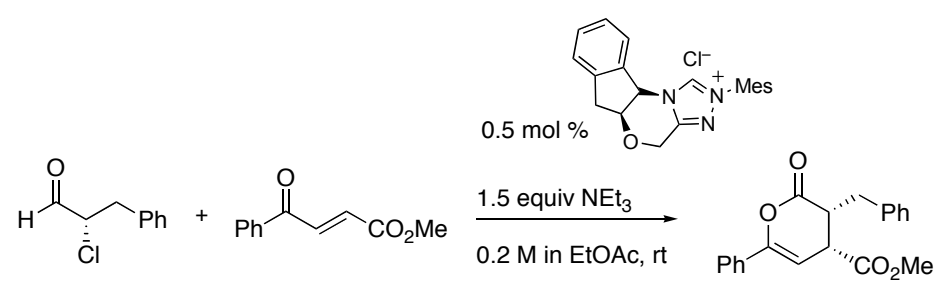

(3R,4R)-methyl 3-benzyl-6-phenyl-2-oxo-3,4-dihydro-2H-pyran-4-carboxylate was prepared according to the general procedure, using enantioenriched 2-chloro-hydrocinnamaldehyde (97\% ee, $110.2 \mathrm{mg}, 0.65 \mathrm{mmol}, 1.2$ equiv), (E)-methyl 4-oxo-4-phenylbut-2-enoate (103.5 mg, 0.54 mmol, 1.0 equiv), triazolium salt ent-1 (1.0 mg, $0.5 \mathrm{~mol} \%)$ and $\mathrm{NEt}_{3}(112 \mu \mathrm{L}, 0.82 \mathrm{mmol}, 1.5$ equiv). The desired dihydropyranone product was obtained as a white solid (120 mg, 70\% yield, $4.5: 1 \mathrm{dr}, 98 \%$ ee $(3 R, 4 R))$.
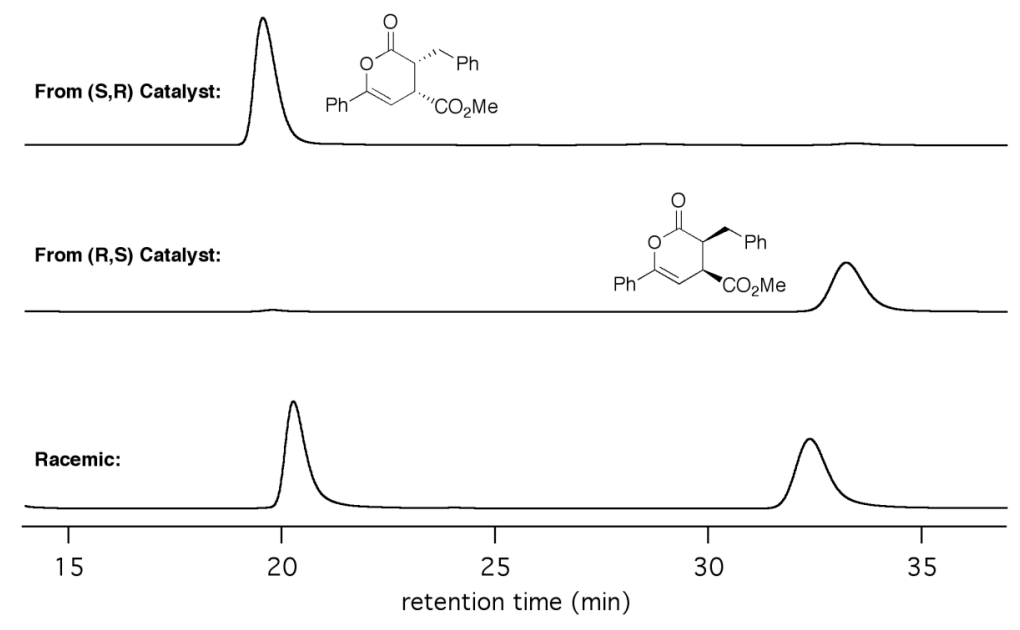

Page S21 


\section{Determination of the enantiomeric excess of the recovered 2-chloro-hydrocinnamaldehyde.}

The same two reactions were set up as above except that the reactions were quenched by the addition of water after $1.5 \mathrm{~h}$ before the reaction had gone to completion. After the same workup, the crude mixture was reduced with $\mathrm{NaBH}_{4}$ in $\mathrm{MeOH}$. The alcohol arising from unreacted chloroaldehyde was isolated and the \%ee was determined by HPLC (AS-H, 100:1 hexanes/ethanol). ${ }^{6}$ With either enantiomer of the triazolium salt, the resulting alcohol was racemic, $t_{1}=20.3 \mathrm{~min}, t_{2}=22.9 \mathrm{~min}$.

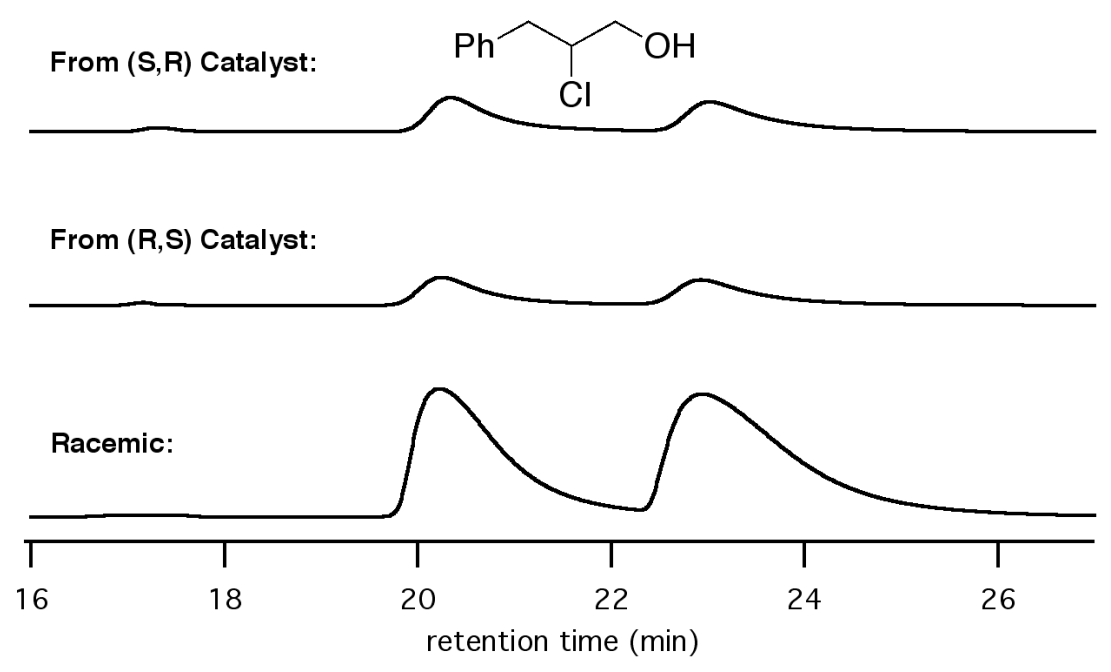

\section{Determination of the relative and absolute stereochemistry of the dihydropyran-2-one products.}

The stereochemistry was determined by single crystal x-ray analysis of the product derived from (E)-methyl 4-oxo-4-(4-bromophenyl)but-2-enoate (Table 1, entry 4): A colorless crystal of approximate dimensions $0.3 * 0.08 * 0.05 \mathrm{~mm}$ was mounted on a glass fiber and transferred to a Bruker CCD platform diffractometer. The $\mathrm{SMART}^{7}$ program was used to determine the unit cell parameters and data collection (20 sec / frame, $0.3 \mathrm{deg}$. /frame for a sphere of diffraction data).

(6) See the supporting information of ref. 2 for the use of this method to determine the enantiomeric excess of this chloroaldehyde.

(7) SMART Software Users Guide, Version 5.1, Bruker Analytical X-ray Systems, Inc., Madison, WI 1999. 
The data were collected at room temperature. The raw frame data were processed using SAINT $^{8}$ program. The absorption correction was applied using program SADABS. ${ }^{9}$ The structure was solved by direct methods and refined on F2 by full-matrix least-squares techniques. Hydrogen atoms were theoretically added. At convergence, $\mathrm{GOF}=1.003$ for 292 variables refined to $\mathrm{R} 1=$ 0.0423 for 2242 reflections with $\mathrm{I}>2 \sigma(\mathrm{I})$. The absolute structure was determined from the Flack parameters, ${ }^{10}$ which are $-0.038(14)$ and $1.035(23)$, respectively. See the corresponding CIF file for further information.

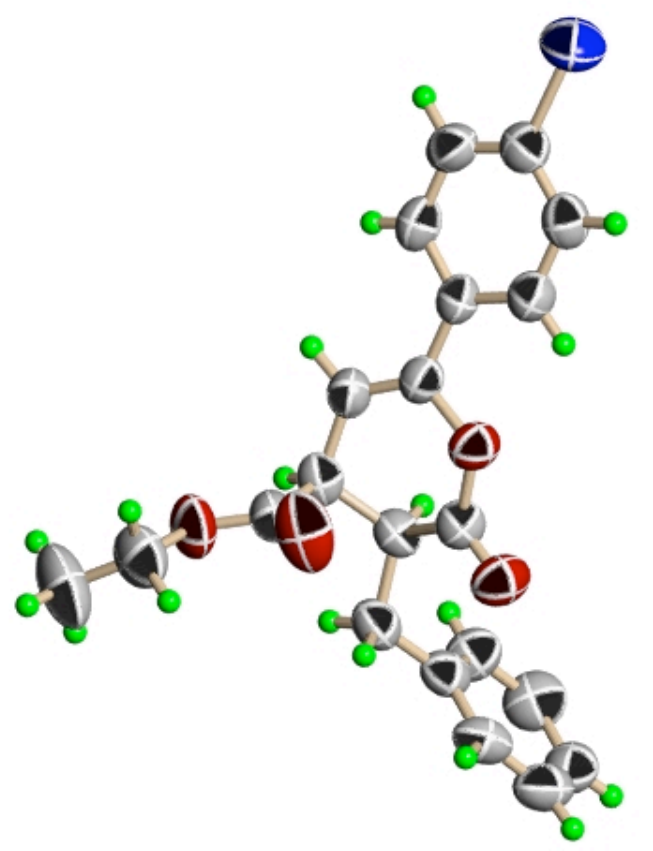

Figure 1. ORTEP representation (50\% probability level) of $(3 S, 4 S)$-methyl 3-benzyl-6-(4bromophenyl)-2-oxo-3,4-dihydro-2H-pyran-4-carboxylate.

(8) SAINT Software Users Guide, Version 5.1, Bruker Analytical X-ray Systems, Inc., Madison, WI 1999.

(9) Sheldrick, G. M. SADABS, Version 2.05, Bruker Analytical X-ray Systerms, Inc.; Madison, WI 2001.

(10) Flack, H. D., Acta Cryst. A39(1983) 876-881. 